\title{
Kinetic dominance and psi series in the Hamilton-Jacobi formulation of inflaton models
}

\author{
Elena Medina $\odot^{*}$ \\ Departamento de Matemáticas, Facultad de Ciencias, Universidad de Cádiz, 11510 Puerto Real, Spain \\ Luis Martínez Alonso ${ }^{\dagger}$ \\ Departamento de Física Teórica II, Facultad de Ciencias Físicas, Universidad Complutense, \\ 28040 Madrid, Spain
}

(Received 20 August 2020; accepted 9 October 2020; published 13 November 2020)

\begin{abstract}
Single-field inflaton models in the kinetic dominance period admit formal solutions given by generalized asymptotic expansions called psi series. We present a method for computing psi series for the Hubble parameter as a function of the inflaton field in the Hamilton-Jacobi formulation of inflaton models. Similar psi series for the scale factor, the conformal time, and the Hubble radius are also derived. They are applied to determine the value of the inflaton field when the inflation period starts and to estimate the contribution of the kinetic dominance period to calculate the duration of inflation. These psi series are also used to obtain explicit two-term truncated psi series near the singularity for the potentials of the Mukhanov-Sasaki equation for curvature and tensor perturbations. The method is illustrated with wide families of inflaton models determined by potential functions combining polynomial and exponential functions, as well as with generalized Starobinsky models.
\end{abstract}

DOI: 10.1103/PhysRevD.102.103517

\section{INTRODUCTION}

The theory of inflationary cosmology provides a framework to study the early Universe [1-3] which solves several central problems of the hot big bang model. In the present work, we consider single-field inflaton models formulated in terms of a time-dependent real field $\phi(t)$ in a spatially flat universe [4-7]. For a homogeneous, spatially flat Friedman-Lemaître-Robertson-Walker (FLRW) spacetime with scale factor $a(t)$, these models are described by the nonlinear ordinary second-order differential equation

$$
\ddot{\phi}+3 H \dot{\phi}+\frac{d V}{d \phi}(\phi)=0
$$

where $H=\dot{a} / a$ is the Hubble parameter, which is related to the inflaton field by

$$
H^{2}=\frac{1}{3 m_{\mathrm{Pl}}^{2}}\left(\frac{1}{2} \dot{\phi}^{2}+V(\phi)\right)
$$

Here $V=V(\phi)$ is a given potential function, $m_{\mathrm{Pl}}=$ $\sqrt{\hbar c / 8 \pi G}$ is the Planck mass, and dots indicate derivatives with respect to the cosmic time $t$.

\footnotetext{
*elena.medina@uca.es

†luism@ucm.es
}

We concentrate on the kinetic dominance (KD) period [8-10] of inflaton models, when the kinetic energy of the inflaton field dominates over its potential energy:

$$
\dot{\phi}^{2} \gg V(\phi) \text {. }
$$

It is a noninflationary or preinflationary stage that is followed by a short fast-roll inflation phase [11] and afterwards by the traditional slow-roll inflation stage [5,12-19]. Recently, Handley et al. [9,10,20-22] have shown the relevance of the KD period [Eq. (3)] for setting initial conditions. In fact, as they prove in Ref. [9], under mild conditions on the potential $V$, all solutions (except perhaps a single one) evolve from a KD region. Our study is devoted to the asymptotic series solutions of the inflaton equations for the KD period and their applications.

The solutions of Eq. (1) manifest generically branch point singularities of logarithmic type. This is the same type of singularity exhibited by the solutions of the Lorentz system [23], and their presence is associated with the socalled psi-series [24] asymptotic solutions of ordinary differential equations. Alternative different psi series containing terms with irrational or even complex exponents were found in the Hénon-Heiles system [25], the rigid body problem, the Toda lattice equation, the Duffing oscillator [26], and the fractional Ginzburg-Landau equation [27]. Logarithmic psi-series solutions of the inflaton equations [Eq. (1)] have been also considered; see, for instance, 
Refs. [8,9,11]. Recently [10], a general method has been formulated for computing psi-series expansions for the solutions of the Eq. (1) and the generalization of Eq. (2) for FLRW spacetimes with curvature. The method formulates the inflaton equations as a four-dimensional first-order system of ordinary differential equations and determines solutions as series expansions involving powers of $t-t^{*}$ and $\log \left(t-t^{*}\right)$. These series are termed logolinear series in Ref. [10].

In the present work, we propose an alternative method for determining formal asymptotic solutions of Eqs. (1) and (2) expressed as psi series. It uses the Hamilton-Jacobi formalism of the inflaton models of Refs. [28,29], and [30], in which the independent variable is the inflaton field instead of the cosmic time. We generate psi series for solutions of the Hamilton-Jacobi equations for the Hubble function as functions of the rescaled inflaton field $\varphi \equiv \sqrt{\frac{3}{2}} \phi / m_{\mathrm{Pl}}$. We apply our method to the following classes of models:

(1)

$$
v(\varphi)=\sum_{n=0}^{N} v_{n}(\varphi) e^{-n \varphi},
$$

where $v_{n}(\varphi)$ are polynomials in $\varphi$, and $N$ is a nonnegative integer.

(2) Models with generalized Starobinsky potential functions,

$$
v(\varphi)=\sum_{n=0}^{N} v_{n} e^{-\alpha n \varphi},
$$

where $\alpha$ is any irrational number and $v_{n}$ are constant coefficients.

In Eqs. (4) and (5), $v(\varphi)$ stands for the rescaled potential $v(\varphi)=3 V(\phi) / m_{\mathrm{Pl}}^{2}$.

The paper is organized as follows: In Sec. II, we briefly introduce the Hamilton-Jacobi formalism of inflation models. Section III describes our method for determining psi series for the inflaton models with potential functions (4) and (5). For the case of Eq. (4), we determine a oneparameter family of logarithmic psi-series solutions in the variable $u:=e^{-\varphi}$ with polynomial coefficients depending on $\varphi$, which could be termed expolinear series. In particular, the models with polynomial potentials $(N=0)$ exhibit several interesting symmetry properties, which are analyzed in detail. As illustrative explicit examples we apply the method to the quadratic potential $v(\varphi)=\mathrm{m}^{2} \varphi^{2}$ and to the Higgs potential $v(\varphi)=g^{2}\left(\varphi^{2}-\lambda^{2}\right)^{2}$. For the models with potential functions [Eq. (5)], we characterize a one-parameter family of psi-series solutions of nonlogarithmic type in the variable $u:=e^{-\varphi}$ with coefficients which are polynomials in $e^{-\alpha \varphi}$. Furthermore, a simple limit operation shows that the results also apply to rational exponents $\alpha$. In particular, we apply the method to the Starobinsky potential

$$
v(\varphi)=\lambda\left(1-e^{-\alpha \varphi}\right)^{2}
$$

and check that our results with $\alpha= \pm \frac{2}{3}$ coincide with the results in Ref. [10] for the corresponding inflaton model with potential

$$
V(\phi)=\Lambda^{2}\left(1-e^{-\sqrt{\frac{2}{3}} \phi}\right)^{2} .
$$

At the end of Sec. III, we discuss how to derive from our psi series in the inflaton field $\varphi$ the logolinear series involving powers of $t-t^{*}$ and $\log \left(t-t^{*}\right)$.

Finally, Sec. IV presents several applications of the psi series obtained in the previous section to calculate analytical approximations of several relevant quantities of inflation models and to compare them with the corresponding numerical approximations. Thus, we use the psi series to determine the value of the inflaton field at the initial moment of the inflation period. We also provide a formula for the amount of inflation, which includes the contribution of the part of the KD period which overlaps the inflation region. Finally, we consider the potentials of the MukanovSasaki equation near the singularity for both curvature and tensor perturbations. It is known [11] that as functions of the conformal time, the dominant terms of these potentials coincide with the critical central singular attractive potential, allowing the fall to the center of a quantum particle. Then, we use the psi series previously obtained to provide an explicit two-term truncated psi-series approximation to these potentials.

\section{HAMILTON-JACOBI FORMULATION OF INFLATON MODELS}

From Eqs. (1) and (2), it follows that

$$
\dot{H}=-\frac{1}{2 m_{\mathrm{Pl}}^{2}} \dot{\phi}^{2} .
$$

As a consequence, the Hubble parameter $H$ is a positive, monotonically decreasing function of $t$. This property implies that for smooth and positive potential functions $V$, the solutions $\phi(t)$ of Eq. (1) with arbitrary finite initial data do not have singularities forward in the cosmic time $t$. Nevertheless, the function $H(t)$ increases without bound backwards in time, so that $H(t)$ and $\phi(t)$ may develop singularities.

The presence of singularities backwards in time can be expected from the following argument: If the KD condition [Eq. (3)] holds, then we may neglect $V$ and $V_{\phi}$ in the inflaton equations, and from Eq. (1) we have 


$$
\ddot{\phi}+\sqrt{\frac{3}{2} \frac{1}{m_{\mathrm{Pl}}}}|\dot{\phi}| \dot{\phi} \sim 0 .
$$

Thus, we obtain two families of approximate solutions:

$$
\phi \sim \pm \sqrt{\frac{2}{3}} m_{\mathrm{Pl}} \log \left(t-t^{*}\right)+\phi_{p} \quad \text { as } t \rightarrow\left(t^{*}\right)^{+}
$$

where $t^{*}$ and $\phi_{p}$ are arbitrary constants. The corresponding asymptotic form of the Hubble parameters is

$$
H \sim \frac{1}{3\left(t-t^{*}\right)} \quad \text { as } t \rightarrow\left(t^{*}\right)^{+} .
$$

These approximate solutions of the inflaton equations are the dominant terms of the psi-series expansions that we will consider below.

\section{A. The Hamilton-Jacobi equations}

We use the rescaled variables

$\varphi=\sqrt{\frac{3}{2} \frac{\phi}{m_{\mathrm{Pl}}},} \quad v(\varphi)=\frac{3}{m_{\mathrm{Pl}}^{2}} V(\phi), \quad h=3 H$

and rewrite Eqs. (1) and (2) as

$$
\ddot{\varphi}+h \dot{\varphi}+\frac{1}{2} v^{\prime}(\varphi)=0
$$

and

$$
h^{2}=\dot{\varphi}^{2}+v(\varphi)
$$

respectively.

In order to discuss inflaton models in the HamiltonJacobi formalism, we consider a reduced space of initial conditions $(\varphi, \dot{\varphi}) \in \mathbb{R}^{2}$ for Eqs. (12) and (13) such that

$$
\varphi \geq \varphi_{0}, \quad \dot{\varphi}<0
$$

where $\varphi_{0}$ will be assumed to be a fixed value of $\varphi$ such that the potential $v$ and its first derivative $v^{\prime}$ are smooth and strictly positive for $\varphi \geq \varphi_{0}$. The map $(\varphi, \dot{\varphi}) \mapsto(\varphi, h)$ enables us to describe the dynamics of Eqs. (12) and (13) on the subset

$$
R=\left\{(\varphi, h) \in \mathbb{R}^{2}: \varphi \geq \varphi_{0}, \sqrt{v(\varphi)}<h<+\infty\right\}
$$

of the $(\varphi, h)$ plane. The Hamilton-Jacobi formulation of Eqs. (1) and (2) is given by the couple of equations

$$
h^{\prime}(\varphi)^{2}=h(\varphi)^{2}-v(\varphi)
$$

and

$$
\dot{\varphi}=-h^{\prime}(\varphi)
$$

Here, primes denote derivatives with respect to $\varphi$, and the Hubble function $h$ is assumed to be the positive root

$$
h=\left(\dot{\varphi}^{2}+v(\varphi)\right)^{1 / 2} .
$$

The set $R$ plays the role of the phase space of the formalism. Each solution $h=h(\varphi)$ of Eq. (16) determines a corresponding implicit solution $\varphi(t)$ of Eq. (17) given by

$$
t=-\int_{\varphi(0)}^{\varphi(t)} \frac{d \varphi}{h^{\prime}(\varphi)}
$$

From the physical point of view, the early Universe is assumed to emerge from a state with energy density $3 m_{\mathrm{Pl}}^{2} H$ of the same order of the Planck density $m_{\mathrm{Pl}}^{4}$. Below that density, the classical inflationary description of the Universe is not valid. Hence, the only physical constraint required for the initial data of a classical inflationary universe is that its energy density $3 m_{\mathrm{Pl}}^{2} H$ should not be larger than $m_{\mathrm{Pl}}^{4}$ or, equivalently, $h \leq m_{\mathrm{Pl}}^{2}$, which in Planck units $(G=c=\hbar=1)$ means

$$
h<h_{p}:=\frac{1}{8 \pi} \approx 0.0398 \text {. }
$$

\section{B. Inflation and kinetic dominance regions}

The inflation period of the Universe evolution is characterized by an accelerated Universe expansion $\ddot{a}>0$. From the identity

$$
\frac{\ddot{a}}{a}=\frac{1}{3 m_{\mathrm{Pl}}^{2}}\left(V(\phi)-\dot{\phi}^{2}\right),
$$

it follows that this period is determined by the constraint

$$
\dot{\phi}^{2}<V(\phi) \text {. }
$$

Then it follows at once that the inflation region [Eq. (22)] in $R$ is characterized by

$$
\sqrt{v}<h<\sqrt{\frac{3 v}{2}} .
$$

For a successful solution to the horizon and flatness cosmological problems, it is required that the amount of inflation during the period of inflation,

$$
N=\int_{\phi_{\text {in }}}^{\phi_{\text {end }}} \frac{H}{\dot{\phi}} d \phi=\frac{1}{3} \int_{\varphi_{\text {end }}}^{\varphi_{\text {in }}} \frac{h}{h^{\prime}} d \varphi,
$$

be close to $N \sim 60[6,7,31,32]$. Thus, given a solution $h=h(\varphi)$ of Eq. (16), it is important to determine the 
values $\varphi_{\text {in }}, \varphi_{\text {end }}$ for which inflation starts and ends, respectively. Due to Eq. (23), both values satisfy

$$
h(\varphi)=\sqrt{\frac{3 v(\varphi)}{2}} .
$$

For instance, in Fig. 1 we plot the reduced Hubble parameter for the quadratic model $v(\varphi)=\mathrm{m}^{2} \varphi^{2}$ together with the functions $\sqrt{v(\varphi)}=\mathrm{m} \varphi$ and $\sqrt{\frac{3 v(\varphi)}{2}}=\sqrt{\frac{3}{2}} \mathrm{~m} \varphi$. Figure 2 shows the same solution entering the inflation region in a $\mathrm{KD}$ regime and leaving the inflation region.

The approximate solutions [Eq. (9)] of Eq. (1) correspond to approximate solutions of Eq. (16) of the form

$$
h \sim \frac{e^{ \pm \varphi}}{b} \quad \text { as } \varphi \rightarrow \pm \infty,
$$

where $b$ is an arbitrary strictly positive parameter. Due to the symmetry $(v(\varphi), h(\varphi), \varphi(t)) \rightarrow(v(-\varphi), h(-\varphi),-\varphi(t))$ of Eqs. (16) and (17), without loss of generality we restrict our analysis to series expansions of solutions of Eq. (16) such that

$$
h \sim \frac{e^{\varphi}}{b} \quad \text { as } \varphi \rightarrow+\infty
$$

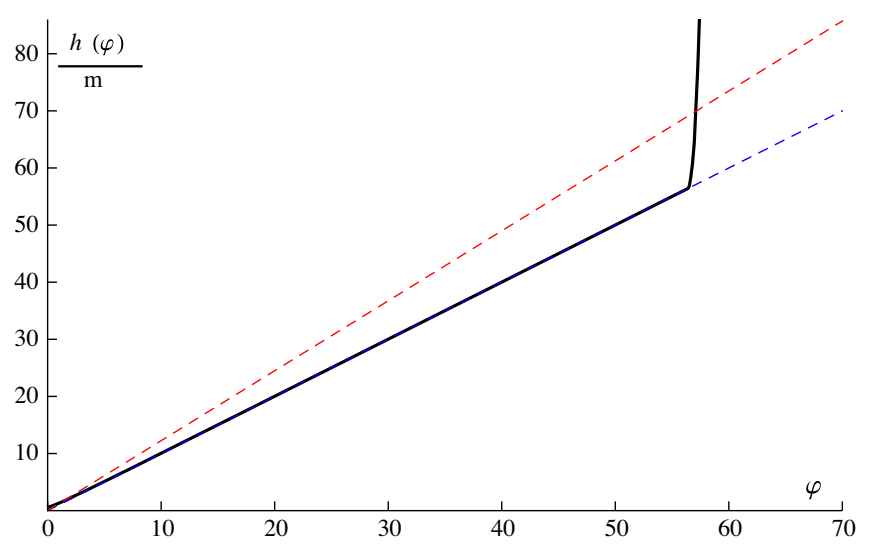

FIG. 1. Reduced Hubble parameter for the quadratic model $v(\varphi)=\mathrm{m}^{2} \varphi^{2}$. The black line shows the rescaled numerical solution $h / \mathrm{m}=h(\varphi) / \mathrm{m}$ of Eq. (16) such that $h(60)=10^{3} \mathrm{~m}$. The region between the blue and red dotted lines is the inflation region.

Solutions $h=h(\varphi)$ of Eq. (16) which have the asymptotic form of Eq. (27) emerge from the KD region and blow up at a finite time $t=t^{*}$ given by

$$
t^{*}=-\int_{\varphi(0)}^{\infty} \frac{d \varphi}{h^{\prime}(\varphi)} .
$$

\section{PSI SERIES IN THE HAMILTON-JACOBI FORMULATION}

In this section, we determine a one-parameter family of psi-series formal solutions of Eq. (16) for the models with polynomial-exponential potentials [Eq. (4)] and Starobinsky potentials [Eq. (5)].

\section{A. Psi series for polynomial-exponential potentials}

Let us consider the differential equation (16) for a potential $v(\varphi)$ of the family in Eq. (4). We look for psiseries solutions of the form

$$
h(\varphi)=\frac{e^{\varphi}}{b}+\sum_{n=1}^{\infty} h_{n}(\varphi) e^{-(n-1) \varphi},
$$

where the coefficients $h_{n}(\varphi)$ are polynomial functions of $\varphi$, and $b$ is a nonzero positive real parameter.

By substituting Eq. (29) into Eq. (16), we obtain

$$
\begin{aligned}
\frac{2}{b} \sum_{n=1}^{\infty} & \left(h_{n}^{\prime}-n h_{n}\right) e^{-(n-2) \varphi}+\sum_{n=0}^{N} v_{n}(\varphi) e^{-n \varphi} \\
= & -\sum_{n=2}^{\infty}\left[\sum_{j+k=n}\left(h_{j}^{\prime}-(j-1) h_{j}\right)\left(h_{k}^{\prime}-(k-1) h_{k}\right)\right. \\
& \left.\quad-h_{j} h_{k}\right] e^{-(n-2) \varphi} .
\end{aligned}
$$

Then, identifying the coefficients of $e^{\varphi}$ in Eq. (30), we have that $h_{1}$ must satisfy the equation $h_{1}^{\prime}-h_{1}=0$, whose polynomial solution is

$$
h_{1}(\varphi)=0
$$
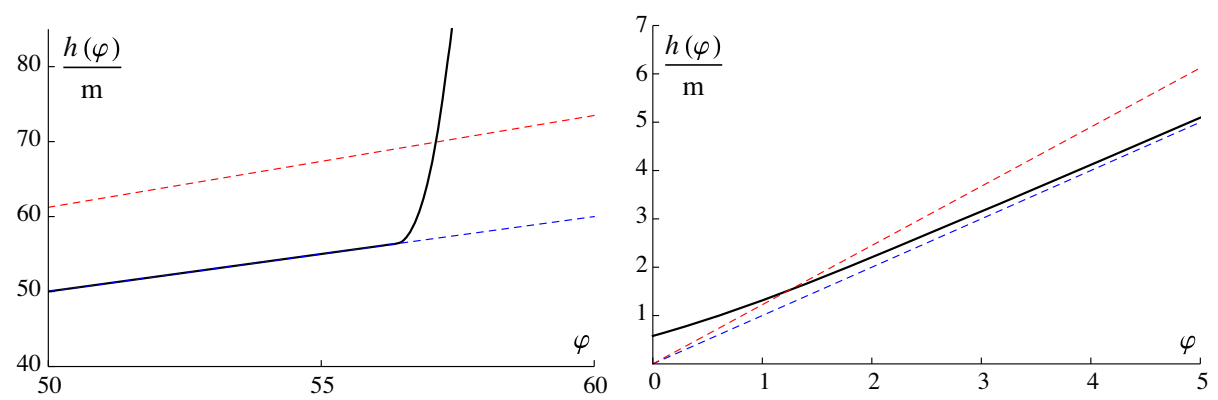

FIG. 2. The left figure shows the solution of Fig. 1 entering the inflation region in a KD regime and approaching the slow-roll regime (blue dotted line). The right figure shows the same solution leaving the inflation region. 
From Eq. (30) and using Eq. (31), it follows that identifying the coefficients of $e^{-n \varphi}$ for $n=0,1, \ldots, N$ implies the recursion relation

$$
h_{n+2}^{\prime}-(n+2) h_{n+2}=-\frac{b}{2}\left[v_{n}+\sum_{j+k=n+2, j, k \geq 2}\left(\left(h_{j}^{\prime}-(j-1) h_{j}\right)\left(h_{k}^{\prime}-(k-1) h_{k}\right)-h_{j} h_{k}\right)\right] .
$$

Furthermore, identifying the coefficients of $e^{-n \varphi}$ for $n>N$ leads to the recursion relation

$$
h_{n+2}^{\prime}-(n+2) h_{n+2}=-\frac{b}{2} \sum_{j+k=n+2, j, k \geq 2}\left(\left(h_{j}^{\prime}-(j-1) h_{j}\right)\left(h_{k}^{\prime}-(k-1) h_{k}\right)-h_{j} h_{k}\right)
$$

The Eqs. (32) and (33) are nonhomogeneous linear ordinary differential equations with constant coefficients for $h_{n+2}$. The nonhomogeneous terms depend on the coefficients $h_{j}$ with $j=2, \ldots, n$ and on the polynomial coefficients $v_{n}(\varphi)$ with $n=0, \ldots, N$ of the potential function in Eq. (4). Therefore, it follows that the coefficients $h_{n}$ of the series in Eq. (29) can be recursively determined as polynomials in $\varphi$. The recursion relations can be formally solved, and they provide us with the polynomial solutions

$$
\begin{aligned}
h_{n+2}(\varphi)= & \frac{b}{2} e^{(n+2) \varphi} \int_{\varphi}^{\infty} e^{-(n+2) s} v_{n}(s) \\
& +\frac{b}{2} e^{(n+2) \varphi} \int_{\varphi}^{\infty} e^{-(n+2) s} \sum_{j+k=n+2, j, k \geq 2}\left(\left(h_{j}^{\prime}(s)-(j-1) h_{j}(s)\right)\left(h_{k}^{\prime}(s)-(k-1) h_{k}(s)\right)-h_{j}(s) h_{k}(s)\right) d s
\end{aligned}
$$

for $n=0,1, \ldots, N$ and

$$
h_{n+2}(\varphi)=\frac{b}{2} e^{(n+2) \varphi} \sum_{j+k=n+2, j, k \geq 2} \int_{\varphi}^{\infty} e^{-(n+2) s}\left(\left(h_{j}^{\prime}(s)-(j-1) h_{j}(s)\right)\left(h_{k}^{\prime}(s)-(k-1) h_{k}(s)\right)-h_{j}(s) h_{k}(s)\right) d s
$$

for $n>N$.

In this way, we have proved that the differential equation (16) for potentials $v(\varphi)$ of the form in Eq. (4) admits a (formal) one-parameter family of psi-series solutions of the form

$$
h(\varphi)=\frac{e^{\varphi}}{b}+\sum_{n=2}^{\infty} h_{n}(\varphi) e^{-(n-1) \varphi} .
$$

The first few equations [Eq. (32)] for $N \geq 2$ are

$$
\begin{aligned}
h_{2}^{\prime}-2 h_{2} & =-\frac{b}{2} v_{0}, \\
h_{3}^{\prime}-3 h_{3} & =-\frac{b}{2} v_{1}, \\
h_{4}^{\prime}-4 h_{4} & =-\frac{b}{2}\left[v_{2}+\left(h_{2}^{\prime}\right)^{2}-2 h_{2} h_{2}^{\prime}\right] .
\end{aligned}
$$

The corresponding solutions [Eq. (34)] are

$$
\begin{aligned}
h_{2}(\varphi)= & \frac{b}{2} e^{2 \varphi} \int_{\varphi}^{\infty} v_{0}(s) e^{-2 s} d s, \\
h_{3}(\varphi)= & \frac{b}{2} e^{3 \varphi} \int_{\varphi}^{\infty} v_{1}(s) e^{-3 s} d s, \\
h_{4}(\varphi) & =\frac{b}{2} e^{4 \varphi} \int_{\varphi}^{\infty} v_{2}(s) e^{-4 s} d s+\frac{b^{3}}{8} e^{4 \varphi} \int_{\varphi}^{\infty} v_{0}(s)^{2} e^{-4 s} d s \\
& -\frac{b^{3}}{4} e^{4 \varphi} \int_{\varphi}^{\infty} e^{-2 s_{1}} v_{0}\left(s_{1}\right)\left(\int_{s_{1}}^{\infty} e^{-2 s_{2}} v_{0}\left(s_{2}\right) d s_{2}\right) d s_{1} .
\end{aligned}
$$

\section{B. Polynomial potentials}

In the polynomial case of Eq. (4), $v(\varphi)=v_{0}(\varphi)$, with $v_{0}(\varphi)$ being a polynomial of degree $d$, the family of psi series [Eq. (36)] reduces to the form

$$
h(\varphi)=\frac{e^{\varphi}}{b}+\sum_{n=1}^{\infty} b^{2 n-1} \gamma_{n}(\varphi) e^{-(2 n-1) \varphi},
$$


where $\gamma_{1}$ is the unique polynomial of degree $d$ which satisfies the equation

$$
\gamma_{1}^{\prime}-2 \gamma_{1}=-\frac{1}{2} v_{0}
$$

and the coefficients $\gamma_{n}(\varphi)(n \geq 2)$ are polynomials of degree $n d-1$, independent of the parameter $b$, which can be recursively determined by

$$
\begin{aligned}
\gamma_{n+1}^{\prime} & -2(n+1) \gamma_{n+1} \\
= & -\frac{1}{2} \sum_{j+k=n+1, j, k \geq 1}\left(( \gamma _ { j } ^ { \prime } - ( 2 j - 1 ) \gamma _ { j } ) \left(\gamma_{k}^{\prime}-(2 k-1)\right.\right. \\
& \left.\left.\times \gamma_{k}\right)-\gamma_{j} \gamma_{k}\right) .
\end{aligned}
$$

Indeed, if we set $n=2 m-1(m \geq 1)$ in Eq. (33), we have that

$$
\begin{aligned}
& h_{2 m+1}^{\prime}-(2 m+1) h_{2 m+1} \\
& =-\frac{b}{2} \sum_{j+k=2 m+1, j, k \geq 2}\left(\left(h_{j}^{\prime}-(j-1) h_{j}\right)\right. \\
& \left.\quad \times\left(h_{k}^{\prime}-(k-1) h_{k}\right)-h_{j} h_{k}\right) .
\end{aligned}
$$

Now, taking into account Eq. (31) and applying induction in $m$, it is clear that

$$
h_{2 m+1} \equiv 0 \quad \text { for all } m \geq 0 \text {. }
$$

In order to make explicit the dependence of $h(\varphi)$ on the arbitrary parameter $b$, we introduce the functions $\gamma_{n}(\varphi):=h_{2 n}(\varphi) / b^{2 n-1}$. Thus, for $n=0$, Eq. (32) reduces to Eq. (40), and Eq. (33) becomes the recursion relation [Eq. (41)]. We notice that the coefficients $\gamma_{n}(\varphi)$ are independent of $b$. The first few equations [Eq. (41)] are

$$
\begin{gathered}
\gamma_{2}^{\prime}-4 \gamma_{2}=-\frac{1}{2}\left[\left(\gamma_{1}^{\prime}\right)^{2}-2 \gamma_{1} \gamma_{1}^{\prime}\right], \\
\gamma_{3}^{\prime}-6 \gamma_{3}=-\left[\gamma_{1}^{\prime} \gamma_{2}^{\prime}-\gamma_{1} \gamma_{2}^{\prime}-3 \gamma_{1}^{\prime} \gamma_{2}+2 \gamma_{1} \gamma_{2}\right], \\
\gamma_{4}^{\prime}-8 \gamma_{4}=-\frac{1}{2}\left[2 \gamma_{1}^{\prime} \gamma_{3}^{\prime}+\left(\gamma_{2}^{\prime}\right)^{2}-2 \gamma_{1} \gamma_{3}^{\prime}-6 \gamma_{2} \gamma_{2}^{\prime}\right. \\
\left.-10 \gamma_{1}^{\prime} \gamma_{3}+8 \gamma_{1} \gamma_{3}+8 \gamma_{2}^{2}\right] .
\end{gathered}
$$

In particular, Eqs. (40) and (43) imply the following explicit relations for general polynomial potentials $v_{0}(\varphi)$ :

$$
\gamma_{1}(\varphi)=\frac{1}{2} e^{2 \varphi} \int_{\varphi}^{\infty} e^{-2 s} v_{0}(s) d s
$$

$$
\begin{aligned}
\gamma_{2}(\varphi)= & -\frac{1}{4} e^{4 \varphi} \int_{\varphi}^{\infty} e^{-2 s_{1}} v_{0}\left(s_{1}\right)\left(\int_{s_{1}}^{\infty} e^{-2 s_{2}} v_{0}\left(s_{2}\right) d s_{2}\right) d s_{1} \\
& +\frac{1}{8} e^{4 \varphi} \int_{\varphi}^{\infty} e^{-4 s_{1}} v_{0}\left(s_{1}\right)^{2} d s_{1}
\end{aligned}
$$

From Eq. (40), we have that $\gamma_{1}$ is a polynomial of the same degree $d$ as $v_{0}(\varphi)$. Consequently, the right-hand side of Eq. (43) is a polynomial of degree $2 d-1$, and so is $\gamma_{2}$. Now, since the coefficient of $\gamma_{n} \gamma_{1}$ on the right-hand side of Eq. (41) is given by $-2(n-1)$, which is nonzero for $n \geq 2$, by using induction in $n$, it follows that for $n \geq 2$, the coefficient $\gamma_{n}(\varphi)$ is a polynomial in $\varphi$ of degree $n d-1$.

Next, we discuss two illustrative examples.

\section{The quadratic potential}

For the quadratic potential

$$
v(\varphi)=\mathrm{m}^{2} \varphi^{2},
$$

we have an expansion for $h$ of the form of Eq. (39) with $\gamma_{1}$ satisfying Eq. (40):

$$
\gamma_{1}^{\prime}-2 \gamma_{1}=-\frac{1}{2} \mathrm{~m}^{2} \varphi^{2}
$$

Hence, we obtain

$$
\gamma_{1}(\varphi)=\frac{\mathrm{m}^{2}}{8}\left(1+2 \varphi+2 \varphi^{2}\right),
$$

so that the first two terms of the psi series of $h$ are

$$
h(\varphi)=\frac{e^{\varphi}}{b}+\frac{b \mathrm{~m}^{2}}{8}\left(1+2 \varphi+2 \varphi^{2}\right) e^{-\varphi}+\cdots .
$$

The coefficients $\gamma_{n}(n \geq 2)$ in Eq. (39) are determined by Eq. (41). For example, we obtain

$$
\begin{aligned}
\gamma_{2}(\varphi)= & -\frac{\mathrm{m}^{4}}{1024}\left(5+20 \varphi+40 \varphi^{2}+32 \varphi^{3}\right), \\
\gamma_{3}(\varphi)= & \frac{\mathrm{m}^{6}}{1990656}\left(703+4218 \varphi+12654 \varphi^{2}\right. \\
& \left.+18504 \varphi^{3}+7344 \varphi^{4}-5184 \varphi^{5}\right) .
\end{aligned}
$$

\section{The Higgs potential}

The KD period for the inflaton model with a Higgs potential

$$
v(\varphi)=g^{2}\left(\varphi^{2}-\lambda^{2}\right)^{2}
$$

has been studied in Ref. [11], where approximate expressions for the inflaton field and the Hubble parameter as functions of $t$ have been obtained. According to our general result for models with polynomial potentials, the Higgs 
model admits a psi series of the form in Eq. (39). In particular, one easily finds that the first coefficients of this psi series are given by

$$
\begin{aligned}
& \gamma_{1}(\varphi)=\frac{g^{2}}{8}\left[3+6 \varphi+6 \varphi^{2}+4 \varphi^{3}+2 \varphi^{4}-2 \lambda^{2}\left(1+2 \varphi+2 \varphi^{2}\right)+2 \lambda^{4}\right], \\
\gamma_{2}(\varphi)= & \frac{g^{4}}{16384}\left[\lambda^{6}(1024 \varphi+768)+\lambda^{4}\left(-3072 \varphi^{3}-4864 \varphi^{2}-3968 \varphi-1760\right)\right. \\
+ & \lambda^{2}\left(3072 \varphi^{5}+7424 \varphi^{4}+10496 \varphi^{3}+9408 \varphi^{2}+4704 \varphi+1176\right) \\
& \left.-\left(1024 \varphi^{7}+3328 \varphi^{6}+6528 \varphi^{5}+8928 \varphi^{4}+8928 \varphi^{3}+6696 \varphi^{2}+3348 \varphi+837\right)\right] .
\end{aligned}
$$

\section{Starobinsky's potentials}

We now consider the class of potentials of the form in Eq. (5). It includes as a particular case the Starobinsky model $[1,33,34]$

$$
v(\varphi)=\lambda\left(1-e^{-\alpha \varphi}\right)^{2}
$$

It can be proved [30] that the existence of approximate solutions [Eq. (27)] of Eq. (16) is only possible if

$$
N \alpha>-2 \text {, }
$$

so that we will henceforth assume that Eq. (55) is satisfied.

We look for psi-series solutions of Eq. (16) of the form

$$
h(\varphi)=\frac{e^{\varphi}}{b}+\sum_{n=1}^{\infty} h_{n}(u) e^{-(n-1) \varphi},
$$

where the coefficients $h_{n}$ are polynomials in

$$
u:=e^{-\alpha \varphi} \text {. }
$$

If we substitute the series in Eq. (56) into Eq. (16), we get

$$
\begin{aligned}
\frac{2}{b} \sum_{n=1}^{\infty} & \left(\alpha u h_{n}^{\prime}+n h_{n}\right) e^{-(n-2) \varphi} \\
= & w(u)+\sum_{n=2}^{\infty}\left(\sum_{j+k=n}\left(\alpha u h_{j}^{\prime}+(j-1) h_{j}\right)\right. \\
& \left.\quad \times\left(\alpha u h_{k}^{\prime}+(k-1) h_{k}\right)-h_{j} h_{k}\right) e^{-(n-2) \varphi},
\end{aligned}
$$

where

$$
w(u):=\sum_{n=0}^{N} v_{n} u^{n} .
$$

Since we assume that $\alpha$ is an irrational number, the powers of $u=e^{-\alpha \varphi}$ and the powers of $e^{\varphi}$ are linearly independent functions; consequently, the coefficients of $e^{-k \varphi}$ for $k \geq-1$ on both sides of Eq. (58) must be equal. Then, for $k=-1$ we obtain $\alpha u h_{1}^{\prime}+h_{1}=0$, and since $h_{1}$ is a polynomial in $u$, we have that

$$
h_{1} \equiv 0 \text {. }
$$

Using Eq. (60) for $k=0$, we get

$$
\alpha u h_{2}^{\prime}+2 h_{2}=\frac{b}{2} w(u)
$$

Equation (61) has a polynomial solution of degree $N$. Proceeding in the same way, vanishing the coefficients of $e^{-n \varphi}$ in Eq. (58) with $n \geq 1$, we obtain the recurrence relation

$$
\begin{aligned}
& \alpha u h_{n+2}^{\prime}+(n+2) h_{n+2} \\
& =\frac{b}{2} \sum_{j+k=n+2, j, k \geq 2}\left(\left(\alpha u h_{j}^{\prime}+(j-1) h_{j}\right)\right. \\
& \left.\quad \times\left(\alpha u h_{k}^{\prime}+(k-1) h_{k}\right)-h_{j} h_{k}\right) .
\end{aligned}
$$

From Eqs. (60)-(62), we conclude that all the coefficients $h_{n}(u)$ in Eq. (56) are recursively determined by Eqs. (61) and (62) as polynomials in $u$. Moreover, if we set $n=2 m-1$ in Eq. (62), then from Eq. (60) and applying induction in $m$, it follows immediately that

$$
h_{2 m+1} \equiv 0, \quad m=0,1,2, \ldots
$$

To make explicit the dependence of $h(\varphi)$ on the arbitrary parameter $b$, we introduce the functions

$$
\gamma_{n}(u):=\frac{h_{2 n}(u)}{b^{2 n-1}} .
$$

Thus, Eqs. (61) and (62) reduce to

$$
\alpha u \gamma_{1}^{\prime}+2 \gamma_{1}=\frac{1}{2} w(u)
$$

and

$$
\begin{aligned}
& \alpha u \gamma_{n+1}^{\prime}+2(n+1) \gamma_{n+1} \\
& =\frac{1}{2} \sum_{j+k=n+1, j, k \geq 1}\left(\left(\alpha u \gamma_{j}^{\prime}+(2 j-1) \gamma_{j}\right)\right. \\
& \left.\quad \times\left(\alpha u \gamma_{k}^{\prime}+(2 k-1) \gamma_{k}\right)-\gamma_{j} \gamma_{k}\right),
\end{aligned}
$$


respectively. Here, primes indicate derivatives with respect to $u$.

Applying induction with respect to $n$ in Eq. (65), we easily conclude that $\gamma_{n}(u)$ is a polynomial in $u$ of degree at most $n N$ (in particular, $\gamma_{1}$ is a polynomial of degree $N$ ). Therefore, we have proved the existence of a psi-series expansion of the form

$$
h(\varphi)=\frac{e^{\varphi}}{b}\left(1+\sum_{n=1}^{\infty} b^{2 n} \gamma_{n}\left(e^{-\alpha \varphi}\right) e^{-2 n \varphi}\right) .
$$

For example, we get the following explicit relations for $\alpha>0$ :

$$
\begin{aligned}
\gamma_{1}(u) & =\frac{1}{2 \alpha} u^{-2 / \alpha} \int_{0}^{u} s^{2 / \alpha-1} w(s) d s, \\
\gamma_{2}(u)= & -\frac{1}{4 \alpha^{2}} u^{-4 / \alpha} \int_{0}^{u} s_{1}^{2 / \alpha-1} w\left(s_{1}\right) \\
& \times\left(\int_{0}^{s 1} s_{2}^{2 / \alpha-1} w\left(s_{2}\right) d s_{2}\right) d s_{1} \\
& +\frac{1}{8 \alpha} u^{-4 / \alpha} \int_{0}^{u} s_{1}^{4 / \alpha-1} w\left(s_{1}\right)^{2} d s_{1},
\end{aligned}
$$

For $\alpha<0$, the same expressions hold with the lower limits of the integrals substituted by $\infty$.

\section{The Starobinsky model}

The potential function of the Starobinsky model is given by

$$
v(\varphi)=\lambda\left(1-e^{-\alpha \varphi}\right)^{2}, \quad \alpha>-1 .
$$

Then the corresponding Eq. (64) reduces to

$$
\alpha u \gamma_{1}^{\prime}+2 \gamma_{1}=\frac{\lambda}{2}(1-u)^{2}
$$

and the first two equations [Eq. (65)] are

$$
\begin{aligned}
& \alpha u \gamma_{2}^{\prime}+4 \gamma_{2}=\frac{(\alpha u)^{2}}{2}\left(\gamma_{1}^{\prime}\right)^{2}+\alpha u \gamma_{1} \gamma_{1}^{\prime}, \\
& \alpha u \gamma_{3}^{\prime}+6 \gamma_{3}=(\alpha u)^{2} \gamma_{1}^{\prime} \gamma_{2}^{\prime}+3 \alpha u \gamma_{1}^{\prime} \gamma_{2}+\alpha u \gamma_{1} \gamma_{2}^{\prime}+2 \gamma_{1} \gamma_{2} .
\end{aligned}
$$

Therefore, the first polynomial coefficients of the expansion in Eq. (56) turn out to be given by

$$
\begin{aligned}
\frac{\gamma_{1}(u)}{\lambda}= & \frac{u^{2}}{4(\alpha+1)}-\frac{u}{\alpha+2}+\frac{1}{4}, \\
\frac{\gamma_{2}(u)}{\lambda^{2}}= & \frac{\alpha u^{4}}{32(\alpha+1)^{2}}-\frac{\alpha(2 \alpha+3) u^{3}}{4(\alpha+1)(\alpha+2)(3 \alpha+4)} \\
& +\frac{\alpha(5 \alpha+6) u^{2}}{16(\alpha+1)(\alpha+2)^{2}}-\frac{\alpha u}{4(\alpha+2)(\alpha+4)}, \\
\frac{\gamma_{3}(u)}{\lambda^{3}}= & \frac{\alpha(4 \alpha+1) u^{6}}{384(\alpha+1)^{3}}-\frac{\alpha(4 \alpha+5)\left(9 \alpha^{2}+16 \alpha+4\right) u^{5}}{32(\alpha+1)^{2}(\alpha+2)(3 \alpha+4)(5 \alpha+6)} \\
& +\frac{\alpha\left(162 \alpha^{4}+739 \alpha^{3}+1190 \alpha^{2}+776 \alpha+160\right) u^{4}}{128(\alpha+1)^{2}(\alpha+2)^{2}(2 \alpha+3)(3 \alpha+4)} \\
& -\frac{\alpha\left(42 \alpha^{4}+301 \alpha^{3}+674 \alpha^{2}+584 \alpha+160\right) u^{3}}{48(\alpha+1)(\alpha+2)^{3}(\alpha+4)(3 \alpha+4)} \\
& +\frac{\alpha\left(13 \alpha^{2}+58 \alpha+40\right) u^{2}}{64(\alpha+2)^{2}(\alpha+3)(\alpha+4)}-\frac{\alpha u}{16(\alpha+4)(\alpha+6)} .
\end{aligned}
$$

\section{Logolinear series}

Once we have determined the psi series [Eqs. (36), (39), and (66)] for $h(\varphi)$, then from Eq. (17), a psi series of logolinear type depending on the variable $t$ [8-11] for the inflaton field $\varphi$ and the reduced Hubble parameter $h$ can be derived. Thus, for polynomial potentials, if we insert Eq. (39) into Eq. (17), we get

$\dot{\varphi}=-\frac{e^{\varphi}}{b}-\sum_{n=1}^{\infty} b^{2 n-1}\left(\gamma_{n}^{\prime}(\varphi)-(2 n-1) \gamma_{n}(\varphi)\right) e^{-(2 n-1) \varphi}$.

It can be easily checked that Eq. (72) admits a family of formal psi-series solutions of the form

$$
\varphi(t)=-x+\sum_{n=1}^{\infty} \alpha_{n}(x)\left(t-t^{*}\right)^{2 n}
$$

where

$$
x:=\log \left(\frac{t-t^{*}}{b}\right),
$$

and $\alpha_{n}(x)$ can be recursively determined as polynomials in $x$. Next, we sketch the main ideas of the proof. First, we introduce the polynomials $\theta_{n}(\varphi)$ and their coefficients $\theta_{n, j}$, $j=0, \ldots, n d$ through

$$
\theta_{n}(\varphi):=\gamma_{n}^{\prime}(\varphi)-(2 n-1) \gamma_{n}(\varphi)=\sum_{j=0}^{n d} \theta_{n, j} \varphi^{j}
$$


and then we rewrite Eq. (72) as

$$
\dot{\varphi}=-\frac{e^{\varphi}}{b}-\sum_{n=1}^{\infty} \sum_{j=0}^{n d} \theta_{n, j} \varphi^{j} b^{2 n-1} e^{-(2 n-1) \varphi}
$$

and substitute Eq. (73) into Eq. (75). For the left-hand side, we have

$\dot{\varphi}=-\frac{1}{t-t^{*}}+\sum_{n=1}^{\infty}\left[\alpha_{n}^{\prime}(x)+2 n \alpha_{n}(x)\right]\left(t-t^{*}\right)^{2 n-1}$.

In order to expand the right-hand side of Eq. (75) in odd powers of $\left(t-t^{*}\right)$, we introduce the Bell's polynomials [35] defined through

$$
\exp \left(\sum_{n=1}^{\infty} x_{n} z^{n}\right)=\sum_{n=0}^{\infty} C_{n}\left(x_{1}, \ldots, x_{n}\right) z^{n}
$$

Then we have that

$$
b^{2 n-1} e^{-(2 n-1) \varphi}=\left(t-t^{*}\right)^{2 n-1} \sum_{m=0}^{\infty} C_{m}\left(A_{m, n}(x)\right)\left(t-t^{*}\right)^{2 m},
$$

where we are introducing the vectorial functions

$$
A_{m, n}(x):=-(2 n-1)\left(\alpha_{1}(x), \ldots, \alpha_{m}(x)\right) .
$$

Thus, by substituting Eqs. (76) and (78) into Eq. (75) and equating the coefficients of $\left(t-t^{*}\right)^{2 n-1}$, we obtain the equations

$$
\begin{aligned}
\alpha_{n}^{\prime}+ & (2 n+1) \alpha_{n} \\
= & \alpha_{n}-C_{n}\left(A_{n, 0}\right)-\operatorname{coeff}\left[\sum_{m=1}^{n} \sum_{j=0}^{m d} \sum_{l=0}^{n-m} \theta_{m, j} C_{l}\left(A_{l, m}\right)\right. \\
& \left.\times\left(-x+\sum_{k=1}^{n-1} \alpha_{k} \tau^{2 k}\right)^{j}, \tau^{2(n-m-l)}\right] .
\end{aligned}
$$

The first Eq. (79) takes the form

$$
\alpha_{1}^{\prime}+3 \alpha_{1}=-\theta_{1}(-x)
$$

so that $\alpha_{1}$ can be determined as a polynomial of degree $d$. Then, since Eq. (79) is an ordinary linear differential equation for $\alpha_{n}$ with nonhomogeneous terms depending only on $\alpha_{1}, \ldots, \alpha_{n-1}$, using induction in $n$, it follows that the coefficients $\alpha_{n}(x)$ can be recursively determined as polynomials of degree at most $n d$. For instance, Eq. (79) for $n=2$ is $\alpha_{2}{ }^{\prime}+5 \alpha_{2}=-\frac{\alpha_{1}(x)^{2}}{2}+\alpha_{1}(x)\left[\theta_{1}(-x)-\theta_{1}{ }^{\prime}(-x)\right]-\theta_{2}(-x)$.

Furthermore, by substituting Eq. (73) into Eq. (39), we find an expansion

$$
h(t)=\frac{1}{t-t^{*}}+\sum_{n=1}^{\infty} \beta_{n}(x)\left(t-t^{*}\right)^{2 n-1},
$$

where $\beta_{n}(x)$ are polynomials in $x$ that can be written in terms of $\alpha_{k}, \gamma_{k}, k=1, \ldots, n$. For example, we have that

$\beta_{1}(x)=\alpha_{1}(x)+\gamma_{1}(-x)$,

$\beta_{2}(x)=\alpha_{2}(x)+\frac{\alpha_{1}(x)^{2}}{2}+\alpha_{1}(x)\left[\gamma_{1}^{\prime}(-x)-\gamma_{1}(-x)\right]+\gamma_{2}(-x)$.

The psi series in Eqs. (73) and (80) are the logolinear expansions derived in Ref. [10] for inflaton models with polynomial potentials in a flat universe.

Analogously, for the Starobinsky potentials [Eq. (5)], the replacement of Eq. (66) into Eq. (17) leads us to

$$
\begin{aligned}
\dot{\varphi}= & -\frac{e^{\varphi}}{b}+\sum_{n=1}^{\infty} b^{2 n-1}\left(\alpha e^{-\alpha \varphi} \gamma_{n}^{\prime}\left(e^{-\alpha \varphi}\right)\right. \\
& \left.+(2 n-1) \gamma_{n}\left(e^{-\alpha \varphi}\right)\right) e^{-(2 n-1) \varphi} .
\end{aligned}
$$

It can be proved that Eq. (81) admits a family of psi-series solutions of the form

$$
\varphi(t)=-\log \left(\frac{t-t^{*}}{b}\right)+\sum_{n=1}^{\infty} \alpha_{n}(\sigma)\left(t-t^{*}\right)^{2 n},
$$

where

$$
\sigma:=\left(\frac{t-t^{*}}{b}\right)^{\alpha}
$$

and $\alpha_{n}(\sigma)$ can be recursively determined as polynomials in $\sigma$. We outline the main ideas of the proof. By introducing the polynomials $\theta_{n}(u)$ and their coefficients $\theta_{n, j}$, $j=0, \ldots, n N$ through

$$
\theta_{n}(u):=\alpha u \gamma_{n}^{\prime}(u)+(2 n-1) \gamma_{n}(u)=\sum_{j=0}^{n N} \theta_{n, j} u^{j},
$$

the Eq. (81) takes the form

$$
\dot{\varphi}=-\frac{e^{\varphi}}{b}+\sum_{n=1}^{\infty} \theta_{n, j} b^{2 n-1} e^{-(2 n-1+j \alpha) \varphi} .
$$

The left-hand side of Eq. (84) is expanded in odd powers of $\left(t-t^{*}\right)$ as 


$$
\dot{\varphi}=-\frac{1}{t-t^{*}}+\sum_{n=1}^{\infty}\left(\alpha \sigma \alpha_{n}^{\prime}(\sigma)+2 n \alpha_{n}(\sigma)\right)\left(t-t^{*}\right)^{2 n-1} .
$$

By using the Bell polynomials [Eq. (77)], we can also expand the terms in the series of the right-hand side of Eq. (84) as

$$
\begin{aligned}
& b^{2 n-1} e^{-(2 n-1+j \alpha) \varphi} \\
& \quad=\left(t-t^{*}\right)^{2 n-1} \sigma^{j} \sum_{m=0}^{\infty} C_{m}\left(A_{m, n, j}(\sigma)\right)\left(t-t^{*}\right)^{2 m},
\end{aligned}
$$

where the vectorial polynomials $A_{m, n, j}$ have been defined as

$$
A_{m, n, j}(\sigma):=-(\alpha j+2 n-1)\left(\alpha_{1}(\sigma), \ldots, \alpha_{n}(\sigma)\right) .
$$

Thus, by substituting Eqs. (85) and (86) into Eq. (84), taking into account that as $\alpha$ is an irrational number, the powers of $\sigma$ and the powers of $\left(t-t^{*}\right)$ are linearly independent, then equating the coefficients of $\left(t-t^{*}\right)^{2 n-1}$ in both sides of the equations, we obtain that $\alpha_{n}$ satisfies the differential equation

$$
\begin{aligned}
& a \sigma \alpha_{n}^{\prime}+(2 n+1) \alpha_{n} \\
& \quad=\alpha_{n}-C_{n}\left(A_{n, 0,0}\right)+\sum_{k+m=n, k \geq 1, m \geq 0} \sum_{j=0}^{k N} \theta_{k, j} \sigma^{j} C_{m}\left(A_{m, k, j}(\sigma)\right) .
\end{aligned}
$$

As the right-hand side of Eq. (87) depends only on $\alpha_{j}$, $j=1, \ldots, n-1$, this equation shows that the coefficients $\alpha_{n}(\sigma)$ can be recursively obtained as polynomials of degree at most $n N$. Thus, for instance, we have that the first two equations [Eq. (87)] are

$\alpha \sigma \alpha_{1}^{\prime}+3 \alpha_{1}=\theta_{1}(\sigma)$

$\alpha \sigma \alpha_{2}^{\prime}+5 \alpha_{2}=-\frac{\alpha_{1}^{2}(\sigma)}{2}-\alpha_{1}(\sigma)\left[\alpha \sigma \theta_{1}^{\prime}(\sigma)+\theta_{1}(\sigma)\right]+\theta_{2}(\sigma)$.

Furthermore, the replacement of Eq. (82) into Eq. (66) provides us with the formal psi series for the reduced Hubble parameter,

$$
h(t)=\frac{1}{t-t^{*}}+\sum_{n=1}^{\infty} \beta_{n}(\sigma)\left(t-t^{*}\right)^{2 n-1},
$$

where $\beta_{n}(\sigma)$ are polynomials in $\sigma$ that can be determined in terms of $\alpha_{k}, \gamma_{k}, k=1, \ldots, n$. Thus, for example, we have that $\beta_{1}(\sigma)=\alpha_{1}(\sigma)+\gamma_{1}(\sigma)$,
$\beta_{2}(\sigma)=\alpha_{2}(\sigma)+\frac{\alpha_{1}(\sigma)^{2}}{2}+\gamma_{2}(\sigma)-\left[\alpha \sigma \gamma_{1}^{\prime}(\sigma)+\gamma_{1}(\sigma)\right] \alpha_{1}(\sigma)$.

We notice that for the Starobinsky model [Eq. (69)], the psi series [Eqs. (82) and (88)] correspond to the logolinear expansions for the inflaton model determined in Ref. [10] for a flat universe, $\lambda=\Lambda^{4}$ and $\alpha= \pm \frac{2}{3}$.

The determination of logolinear series for the general class of polynomial exponential potentials [Eq. (4)] is more involved, and together with the logolinear series for some generalizations of the class of Starobinsky potentials [Eq. (5)], will be the subject of a future work.

\section{APPLICATIONS}

Many analytical calculations for inflation models assume the slow-roll approximation of Eqs. (1) and (2):

$$
H^{2} \sim \frac{1}{3 m_{\mathrm{Pl}}^{2}} V, \quad \dot{\phi} \sim-\frac{1}{3 H} \frac{d V}{d \phi},
$$

which in terms of the reduced Hubble function $h(\varphi)$ can be expressed as

$$
h \sim \sqrt{v}, \quad h^{\prime} \sim(\sqrt{v})^{\prime} .
$$

However, for calculations involving contributions of the $\mathrm{KD}$ period, it is more appropriate to use truncations of psi series.

\section{A. The inflation region}

From Eq. (20), it follows that we may estimate the value $\varphi_{\text {class }}(b)$ of the inflaton field at the beginning of the classical period as a function of $b$ by means of the equation

$$
h\left(\varphi_{\text {class }}, b\right)=h_{p}=\frac{1}{8 \pi} .
$$

In particular, since the approximation in Eq. (27) is model independent, we obtain the general estimation

$$
\varphi_{\text {class }} \approx \log \left(\frac{b}{8 \pi}\right)
$$

A standard procedure [6,7] to calculate the boundary values $\varphi_{\text {in }}$ and $\varphi_{\text {end }}$ of the inflation period proceeds as follows: we first determine $\varphi_{\text {end }}$ by imposing $\epsilon_{V}=1$, where

$$
\epsilon_{V}=\frac{m_{\mathrm{Pl}}^{2}}{2}\left(\frac{V_{\phi}}{V}\right)^{2}
$$

is the potential slow-roll parameter. It leads to the equation 


$$
v^{\prime}\left(\varphi_{\mathrm{end}}\right)=\frac{2}{\sqrt{3}} v\left(\varphi_{\mathrm{end}}\right) .
$$

Then $\varphi_{\text {in }}$ is calculated by using the slow-roll approximation during the whole period of inflation and by adjusting the amount of inflation

$$
N \approx \frac{1}{3} \int_{\varphi_{\text {end }}}^{\varphi_{\text {in }}} \frac{\sqrt{v(\varphi)}}{(\sqrt{v(\varphi)})^{\prime}} d \varphi
$$

to $N \sim 60$. This method leads to the same inflation period for all the solutions of the inflaton model, since Eqs. (94) and (95) depend only on the potential $v(\varphi)$. Nevertheless, in general, the inflation period depends on the solution used, since each solution has a different kinetically dominated fraction of its inflation region.

In order to include the effect of the KD part of the inflation period, we may proceed as follows. We first calculate the function $\varphi_{\text {in }}(b)$ by means of the Eq. (25):

$$
h\left(\varphi_{\text {in }}, b\right)=\sqrt{\frac{3 v\left(\varphi_{\text {in }}\right)}{2}},
$$

where $h$ is approximated by a truncation with a given number $n+1$ of terms of the psi series [Eq. (36), (39), or (66)] corresponding to the model. Then, to calculate $N(b)$, we look for a value $\varphi^{*}(b)$ such that the KD approximation holds for the interval $\varphi \in\left(\varphi^{*}(b), \varphi_{\text {in }}(b)\right)$, while the slowroll approximation holds for the remaining part of the inflation period. Thus, we estimate the amount of inflation $N(b)$ for the psi series corresponding to $b$ by

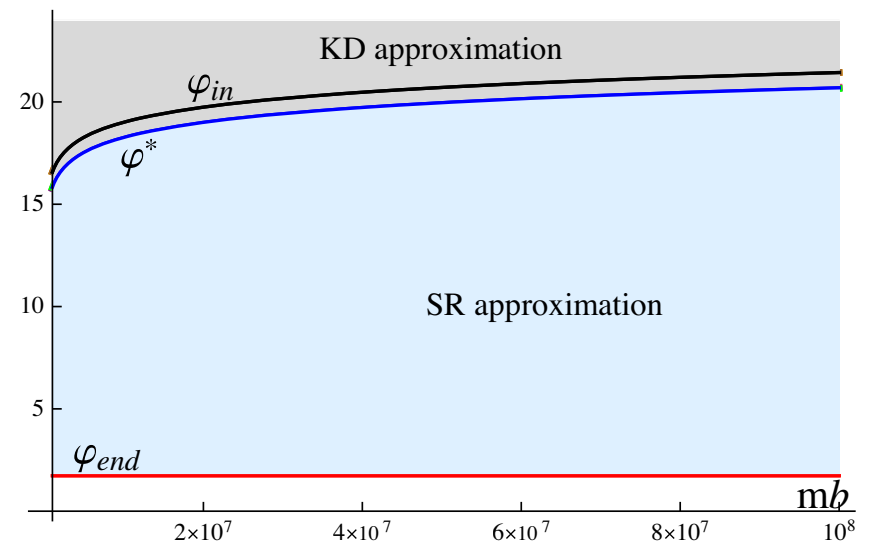

$N(b) \approx \frac{1}{3} \int_{\varphi_{\text {end }}}^{\varphi^{*}(b)} \frac{\sqrt{v(\varphi)}}{(\sqrt{v(\varphi)})^{\prime}} d \varphi+\frac{1}{3} \int_{\varphi^{*}(b)}^{\varphi_{\text {in }}(b)} \frac{h(\varphi, b)}{h_{\varphi}(\varphi, b)} d \varphi$.

The point now is how to get an appropriate value for $\varphi^{*}(b)$. To this end, we observe that the KD approximation means that $h^{\prime} \sim h$ and $h \gg \sqrt{v}$, and that it does not hold as $h^{\prime}(\varphi)$ gets close to zero. Thus, from Eq. (16) we have that the truncated psi series cannot provide a good approximation when $h_{\varphi}(\varphi, b)^{2}$ becomes small. Consequently, we take $\varphi^{(n)}(b)\left[\varphi^{(n)}(b)<\varphi_{\text {in }}(b)\right]$ as the local minimum of $h_{\varphi}(\varphi, b)^{2}$ closest to $\varphi_{\text {in }}(b)$. Numerical evidence shows that the $\mathrm{KD}$ approximation is not acceptable for $\varphi$ very close to $\varphi^{(n)}(b)$, so then $\varphi^{*}(b)$ must be strictly larger than $\varphi^{(n)}(b)$. In order to introduce this correction, we use for $\varphi^{*}(b)$ an expression of the form

$$
\varphi^{*}(b)=\alpha \varphi^{(n)}(b)+\beta \varphi_{\text {in }}(b),
$$

with two real positive parameters $\alpha$ and $\beta$ such that $\alpha+\beta=1$. For example, we have taken $\alpha=0.9$ and $\beta=0.1$ in the examples of Figs. 3-7.

The approximation (97) can be used to select the appropriate value of the parameter $b$, and consequently the initial conditions, corresponding to a solution of Eq. (16) with a previously fixed value of $N(b)$.

Figures 3 and 4 show the application of this method to the quadratic model $v(\varphi)=\mathrm{m}^{2} \varphi^{2}$. We use the truncated psi series with seven terms. It is worthy to point out that very good approximations are also obtained when fewer terms - four, five, or six - are used. We illustrate this fact in Fig. 3 (right), where we use also truncated series with four,

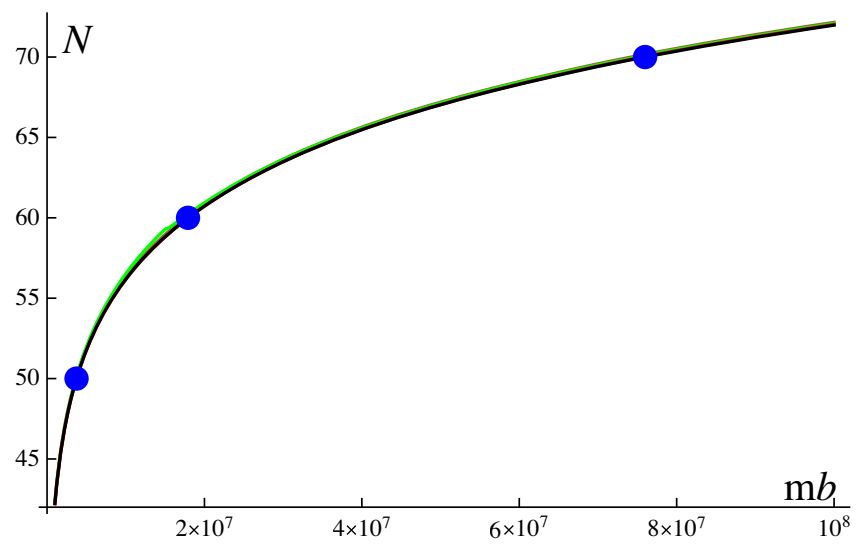

FIG. 3. The left figure shows the lines $\varphi_{\text {in }}(b)$ (black line), $\varphi^{*}(b)$ (blue line), and $\varphi_{\text {end }}$ (red line) for the quadratic model $v(\varphi)=\mathrm{m}^{2} \varphi^{2}$. It also shows the regions in the $(\mathrm{m} b, \varphi)$ plane corresponding to the KD approximation (gray region) and the SR approximation (blue region). The right figure shows the amount of inflation $N(b)$ corresponding to the approximations with 4 (brown line), 5 (green line), 6 (red line) and 7 (black line) terms in the truncated series [Eq. (39)] provided by Eq. (97). The lines are almost completely overlapped, which shows that a very good approximation is obtained with only a few terms of the truncated series. The blue dots indicate the values $N=50,60$, and 70 . 


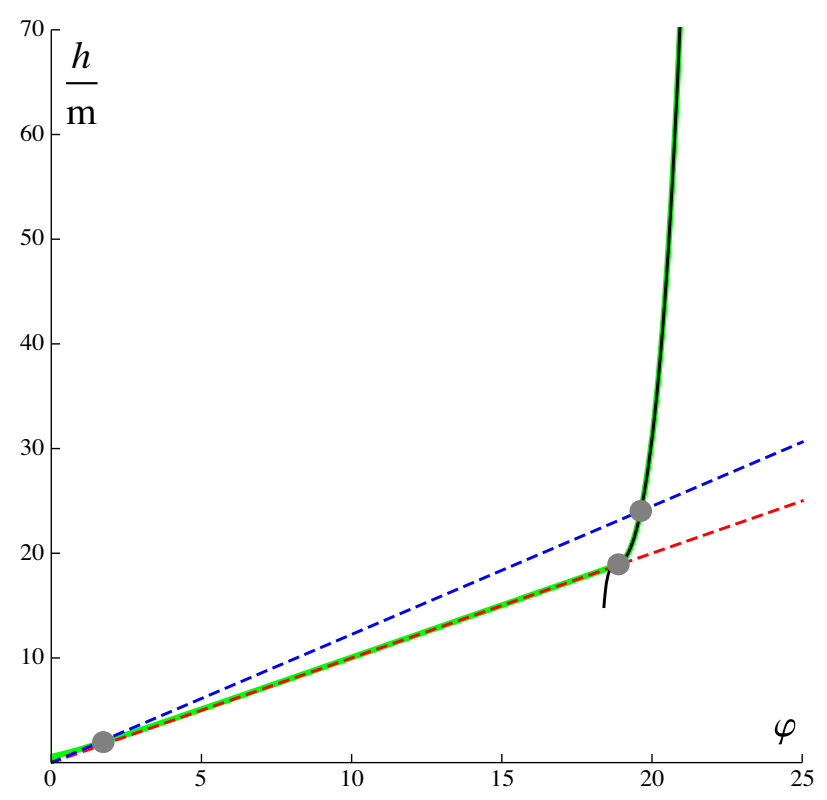

FIG. 4. The green line shows the rescaled numerical solution $h / \mathrm{m}=h(\varphi) / \mathrm{m}$ of Eq. (16) with initial conditions $\left[\varphi_{0}=\right.$ $\left.22, h_{0}=h\left(\varphi_{0}, b_{60}\right) \approx 200.79 \mathrm{~m}\right]$ for the quadratic model $v(\varphi)=\mathrm{m}^{2} \varphi^{2}$. The black line shows the $\mathrm{KD}$ approximation. The region between blue and red dotted lines is the inflation region. The gray dots correspond (from right to left) to $\varphi_{\text {in }}, \varphi^{*}$, and $\varphi_{\text {end }}$. We notice that the black line (KD approximation) overlaps the green line (numerical approximation) for $\varphi \in\left(\varphi^{*}, \varphi_{\text {in }}\right)$, while the red dashed line (SR approximation) overlaps the green line for $\varphi \in\left(\varphi_{\text {end }}, \varphi^{*}\right)$.

five, or six terms. It means that the $\mathrm{KD}$ approximation is rather reliable.

In particular, for $N(b)=60$, we obtain $b_{60}=\frac{\hat{b}_{60}}{\mathrm{~m}}, \hat{b}_{60} \approx$ $1.79104 \times 10^{7}$. In Fig. 4, we plot the numerical solution $h(\varphi)$ of Eq. (16) with initial conditions $\varphi_{0}=22$, $h_{0}=h\left(\varphi_{0}, b_{60}\right)$. Numerical computation of the amount of inflation for this solution leads to $N_{\text {num }} \approx 60.86$. This figure shows how the KD approximation fits accurately the numerical approximation for $\varphi \in\left(\varphi^{*}(b), \varphi_{\text {in }}(b)\right)$.

We may also consider $\varphi_{\text {in }}$ as a function of $N$, and compare it with the SR approximation for $\varphi_{\text {in }}$, which derives from Eq. (95)-i.e.,

$$
\tilde{\varphi}_{\text {in }}(N)=\sqrt{3+6 N}
$$

Figure 5 shows the graphs of both approximations to $\varphi_{\text {in }}(N)$. It can be observed that the result of taking into account the effect of the KD stage leads to greater values of $\varphi_{\text {in }}(N)$.

We apply the same scheme to the Starobinsky model $v(\varphi)=\lambda\left(1-e^{-\frac{\varphi}{\sqrt{3}}}\right)^{2}$, and exhibit the corresponding graphics in Figs. 6 and 7. We use the truncated psi series with seven terms, although we exhibit also in Fig. 6 (right) how the difference with the results corresponding to truncated

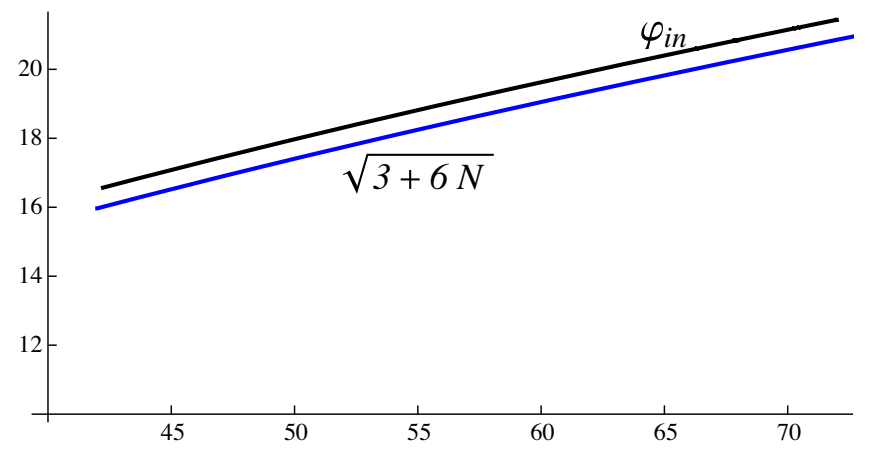

FIG. 5. The black line shows $\varphi_{\text {in }}$ as a function of $N$, where $\varphi_{\text {in }}$ is obtained from Eq. (96) and $N$ from the formula in Eq. (97), which takes into account the contributions of both the KD stage and the SR stage. The blue line shows $\varphi_{\text {in }}$ as a function of $N$ as given by the SR approximation [Eq. (98)].

series with four, five, or six terms is almost imperceptible. In this case, a solution with $N(b)=60$ corresponds to a value $b_{60}=\hat{b}_{60} / \sqrt{\lambda}, \hat{b}_{60} \approx 2649.03$, and the numerical solution with initial condition $\varphi_{0}=12, h_{0}=h\left(\varphi_{0}, b_{60}\right)$ gives the number of e-folds, $N_{\text {num }}(b) \approx 59.5$. Figure 7 shows how the KD approximation fits accurately the numerical approximation for $\varphi \in\left(\varphi^{*}(b), \varphi_{i n}(b)\right)$.

\section{B. The KD period and the Mukhanov-Sasaki equation}

As was shown in Refs. [9-11], truncations of logolinear series expansions involving powers of $t-t^{*}$ and $\log \left(t-t^{*}\right)$ are useful to determine the effect of the KD period on the power spectrum of the primordial curvature and tensor perturbations. These perturbations are characterized by the Mukhanov-Sasaki equation [29]

$\left(\frac{d^{2}}{d \eta^{2}}+k^{2}-W_{\alpha}(\eta)\right) S_{\alpha}(k, \eta)=0 ; \quad \alpha=R, T$,

where the potential $W_{\alpha}(\eta)$ felt by the perturbations is

$$
W_{\alpha}(\eta)=\left\{\begin{array}{l}
\frac{Z_{\eta \eta}}{Z} \text { for curvature perturbations } \\
\frac{a_{\eta \eta}}{a} \text { for tensor perturbations. }
\end{array}\right.
$$

Here $Z=a \dot{\phi} / H$, and $\eta$ is the conformal time defined, up to a constant, by $d t=a d \eta$.

We now apply the psi series depending on $\varphi$ obtained above and several of their consequences to determine approximate expressions for the potentials $W_{\alpha}(\eta)$.

\section{Polynomial-exponential potentials}

For models with a potential $v(\varphi)$ of the form in Eq. (4), we consider the two-term approximation

$$
h(\varphi) \approx e^{\varphi}\left(\frac{1}{b}+h_{2}(\varphi) e^{-2 \varphi}\right)
$$

of the psi series in Eq. (36). The polynomial coefficient $h_{2}(\varphi)$ satisfies 

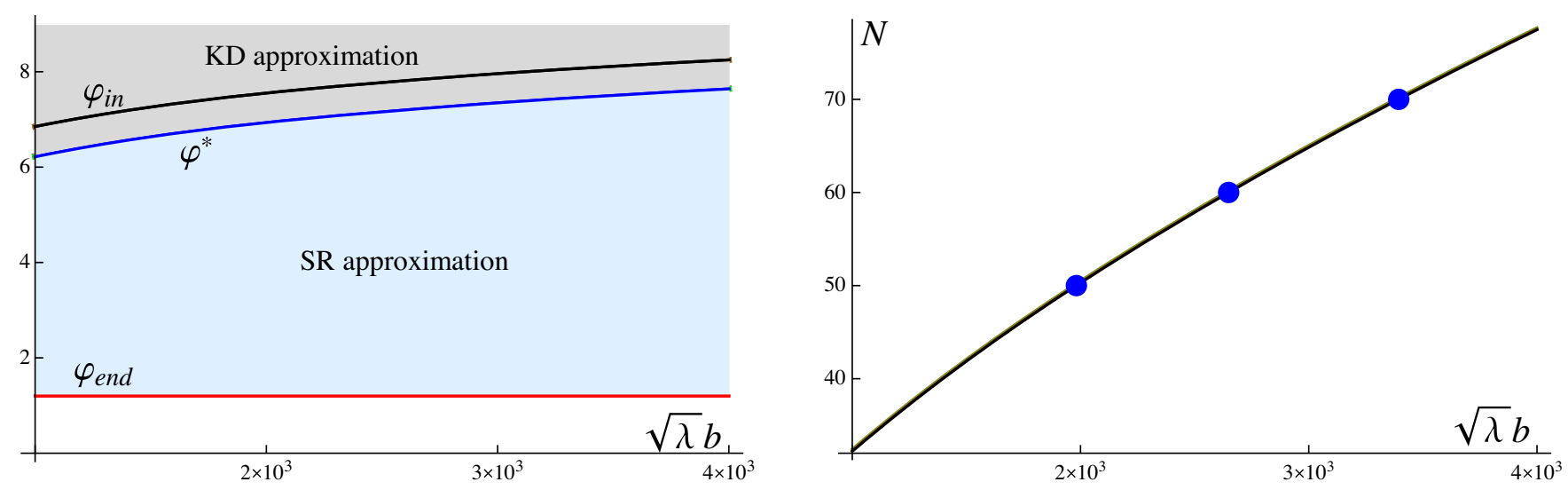

FIG. 6. The left figure shows the lines $\varphi_{\text {in }}(b)$ (black line), $\varphi^{*}(b)$ (blue line), and $\varphi_{\text {end }}$ (red line), as well as the regions in the $(\sqrt{\lambda} b, \varphi)$ plane corresponding to the KD approximation (gray region) and the SR approximation (blue region) for the Starobinsky model $v(\varphi)=\lambda\left(1-e^{-\frac{\varphi}{\sqrt{3}}}\right)^{2}$. The right figure shows the amount of inflation $N(b)$ determined by the approximations [Eq. (97)] with 4 (brown line), 5 (green line), 6 (red line), and 7 (black line) terms in the truncated series [Eq. (66)] provided by Eq. (97). The lines are almost completely overlapped, which shows that a very good approximation is obtained with only a few terms in the truncated series. The blue dots correspond to $N=50,60$, and 70 .

$$
h_{2}^{\prime}-2 h_{2}=-\frac{b}{2} v_{0}
$$

so that it is given by

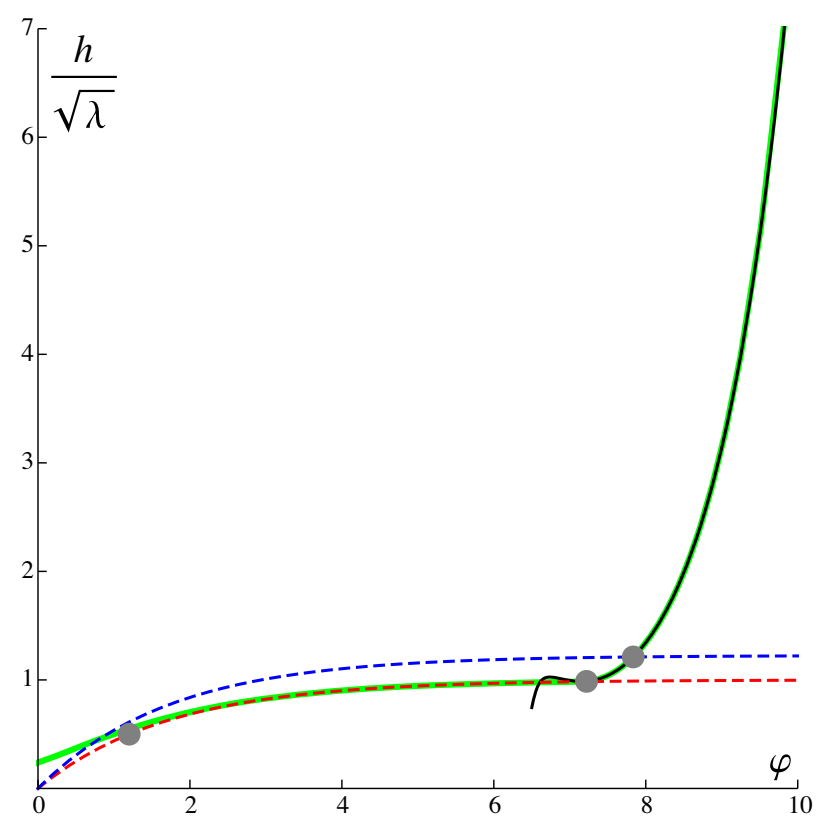

FIG. 7. The green line shows the numerical solution $h / \sqrt{\lambda}=$ $h(\varphi) / \sqrt{\lambda}$ of Eq. (16) with initial conditions $\left[\varphi_{0}=12\right.$, $\left.h_{0}=h\left(\varphi_{0}, b_{60}\right) \approx 61.4435 \sqrt{\lambda}\right]$ for the Starobinsky model $v(\varphi)=\lambda\left(1-e^{-\frac{\varphi}{\sqrt{3}}}\right)^{2}$. The black line shows the KD approximation. The region between the blue and red dotted lines is the inflation region. The gray dots correspond (from right to left) to $\varphi_{\text {in }}, \varphi^{*}$, and $\varphi_{\text {end }}$. It has to be noticed that the black line (KD approximation) overlaps the green line (numerical approximation) for $\varphi \in\left(\varphi^{*}, \varphi_{\text {in }}\right)$, while the red dashed line (SR approximation) overlaps the green line for $\varphi \in\left(\varphi_{\text {end }}, \varphi^{*}\right)$.

$$
h_{2}(\varphi)=\frac{b}{2} e^{2 \varphi} \int_{\varphi}^{+\infty} v_{0}(x) e^{-2 x} d x
$$

We show below several psi series of the form

$$
f(\varphi)=e^{\alpha \varphi}\left(\beta+\sum_{n=2}^{\infty} f_{n}(\varphi) e^{-n \varphi}\right),
$$

with polynomial coefficients $f_{n}(\varphi)$, which are consequences of Eq. (36) and are required for our calculations:

(1) The scale factor admits the expansion

$$
a(\varphi)=c e^{-\frac{\varphi}{3}}\left(1+\sum_{n=2}^{\infty} a_{n}(\varphi) e^{-n \varphi}\right)
$$

where $c$ is an arbitrary nonzero positive constant and

$$
a_{2}(\varphi)=\frac{b}{3} h_{2}(\varphi) .
$$

(2) The conformal time $\eta$, considered as a function of $\varphi$, satisfies the differential equation

$$
\frac{d \eta}{d \varphi}=-\frac{1}{a h^{\prime}},
$$

and then it can be expanded as a psi series

$$
\eta(\varphi)=\frac{e^{-\frac{2 \varphi}{3}}}{c}\left(\frac{3}{2} b+\sum_{n=2}^{\infty} \eta_{n}(\varphi) e^{-n \varphi}\right)
$$


with

$$
\eta_{2}(\varphi)=b^{2} e^{\frac{8}{3} \varphi} \int_{\infty}^{\varphi}\left(h_{2}^{\prime}(x)-\frac{2}{3} h_{2}(x)\right) e^{-\frac{8}{3} x} d x .
$$

(3) The Hubble radius $1 / a H=3 / a h$ can be expanded as

$$
\frac{1}{a H}=\frac{3 b}{c} e^{-\frac{2}{3} \varphi}\left(1+\sum_{n=2}^{\infty} r_{n}(\varphi) e^{-n \varphi}\right)
$$

where

$$
r_{2}(\varphi)=-\frac{4 b}{3} h_{2}(\varphi)
$$

(4) The function $Z=a \dot{\phi} / H$ is proportional to $z(\varphi) \equiv$ $-a h^{\prime} / h$, which has the psi-series expansion

$$
z(\varphi)=-c e^{-\frac{\varphi}{3}}\left(1+\sum_{n=2}^{\infty} z_{n}(\varphi) e^{-n \varphi}\right),
$$

where

$$
z_{2}(\varphi)=b\left(h_{2}^{\prime}(\varphi)-\frac{5}{3} h_{2}(\varphi)\right)
$$

Let us now consider the potentials $W_{\alpha}(\eta)$ [Eq. (100)]. From Eq. (108), we get the following approximation for $\varphi(\eta)$ :

$$
\varphi \approx-\frac{3}{2} \log \left(\frac{2 c}{3 b} \eta\right)
$$

Inserting this result into Eq. (108), we get an approximation with two terms for $\varphi(\eta)$ given by

$\varphi \approx-\frac{3}{2} \log \left(\frac{2 c}{3 b} \eta\right)+\frac{1}{b}\left(\frac{2 c}{3 b} \eta\right)^{3} \eta_{2}\left(-\frac{3}{2} \log \left(\frac{2 c}{3 b} \eta\right)\right)$.

Then, using Eq. (112), we obtain the two-term approximation

$z(\eta) \approx-c\left(\frac{2 c}{3 b} \eta\right)^{1 / 2}\left(1+\tilde{z}_{2}(\log \eta) \eta^{3}\right), \quad \eta \rightarrow 0$,

where

$$
\begin{aligned}
\tilde{z}_{2}(\log \eta) \equiv & \left(\frac{2 c}{3 b}\right)^{3}\left[z_{2}\left(-\frac{3}{2} \log \left(\frac{2 c}{3 b} \eta\right)\right)\right. \\
& \left.-\frac{1}{3 b} \eta_{2}\left(-\frac{3}{2} \log \left(\frac{2 c}{3 b} \eta\right)\right)\right] .
\end{aligned}
$$

Thus, we get that

$$
W_{R}(\eta)=\frac{z_{\eta \eta}}{z} \approx \frac{1}{\eta^{2}}\left[-\frac{1}{4}+\eta^{3}\left(\tilde{z}_{2}^{\prime \prime}(\log \eta)+6 \tilde{z}_{2}^{\prime}(\log \eta)+9 \tilde{z}_{2}(\log \eta)\right)\right], \quad \eta \rightarrow 0
$$

A completely similar calculation starting from Eqs. (105) and (106) leads to

$$
W_{T}(\eta) \approx \frac{1}{\eta^{2}}\left[-\frac{1}{4}+\eta^{3}\left(\tilde{a}_{2}^{\prime \prime}(\log \eta)+6 \tilde{a}_{2}^{\prime}(\log \eta)+9 \tilde{a}_{2}(\log \eta)\right)\right], \quad \eta \rightarrow 0,
$$

with

$$
\tilde{a}_{2}(\log \eta) \equiv\left(\frac{2 c}{3 b}\right)^{3}\left[a_{2}\left(-\frac{3}{2} \log \left(\frac{2 c}{3 b} \eta\right)\right)-\frac{1}{3 b} \eta_{2}\left(-\frac{3}{2} \log \left(\frac{2 c}{3 b} \eta\right)\right)\right]
$$

For example, for the quadratic model in Eq. (48), from Eqs. (49), (106), (109), (113), and (117)-(120) we obtain

$$
\begin{aligned}
& W_{R}(\eta) \approx-\frac{1}{4 \eta^{2}}-\frac{\mathrm{m}^{2} c^{3}}{198 b} \eta\left[131+756 \log \left(\frac{2 c}{3 b} \eta\right)+504 \log \left(\frac{2 c}{3 b} \eta\right)^{2}\right], \\
& W_{T}(\eta) \approx-\frac{1}{4 \eta^{2}}+\frac{\mathrm{m}^{2} c^{3}}{64 b} \eta\left[-1+4 \log \left(\frac{2 c}{3 b} \eta\right)+24 \log \left(\frac{2 c}{3 b} \eta\right)^{2}\right] .
\end{aligned}
$$

These potentials are plotted in Fig. 8, where $b=b_{60}$ is the value obtained in Sec. IV A for the quadratic model and $c$ is taken such that $a\left(\varphi_{\text {in }}\right)=1$. 

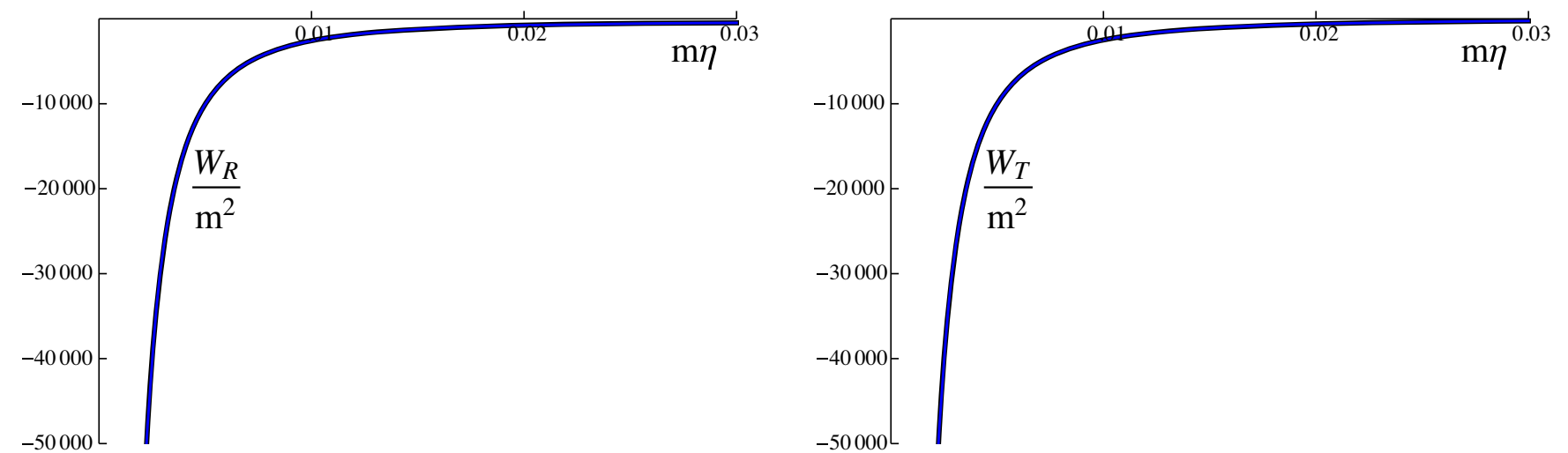

FIG. 8. Potentials $W_{R}(\eta)$ (left) and $W_{T}(\eta)$ (right) for the quadratic model $v(\varphi)=\mathrm{m}^{2} \varphi^{2}$. The black lines show the numerical approximations, while the blue lines show the psi-series approximation [Eq. (121)]. Here we have used the rescaled variables $\mathrm{m} \eta, \mathrm{mm}^{-2} W_{R}, \mathrm{~m}^{-2} W_{T}$, the value $b=b_{60}=\hat{b}_{60} / \mathrm{m}, \hat{b}_{60} \approx 1.79104 \times 10^{7}$ obtained in Sec. IV A for the quadratic model, and $c$ is such that $a\left(\varphi_{\text {in }}\right)=1$. We have taken a value $\eta\left(t^{*}\right)=0$ of the conformal time in agreement with Eq. (108).

\section{Starobinsky potentials}

with $a_{n}(u)$ being polynomials in $u$. In particular,

For models with a potential $v(\varphi)$ of the form in Eq. (5), we consider the psi series in Eq. (66) for $h(\varphi)$ and its truncation to two terms:

$$
a_{1}(u)=\frac{1}{3} \gamma_{1}(u)
$$

$$
h(\varphi) \approx \frac{e^{\varphi}}{b}\left(1+b^{2} \gamma_{1}\left(e^{-\alpha \varphi}\right) e^{-2 \varphi}\right),
$$

where $\gamma_{1}$ is given by Eq. (67) for $\alpha>0$ and by setting the lower integration index as $\infty$ for $\alpha \in\left(-\frac{2}{N}, 0\right)$. From Eq. (66), we derive the psi series which follow:

(1) The scale factor

$$
a(\varphi)=c e^{-\frac{\varphi}{3}}\left(1+\sum_{n=1}^{\infty} b^{2 n} a_{n}(u) e^{-2 n \varphi}\right),
$$

(2) The conformal time

$$
\eta(\varphi)=\frac{3 b}{2 c} e^{-\frac{2 \varphi}{3}}\left(1+\sum_{n=1}^{\infty} b^{2 n} \eta_{n}(u) e^{-2 n \varphi}\right),
$$

where $\eta_{n}(u)$ are polynomials in $u$. For example, we have that

$$
\eta_{1}(u)=\left\{\begin{array}{lc}
\frac{2}{3} \int_{0}^{u}\left(\gamma_{1}^{\prime}(\mu)+\frac{2}{3 \alpha \mu} \gamma_{1}(\mu)\right)\left(\frac{\mu}{u}\right)^{\frac{8}{3 \alpha}} d \mu, & \alpha>0, \\
-\frac{2}{3} \int_{u}^{\infty}\left(\gamma_{1}^{\prime}(\mu)+\frac{2}{3 \alpha \mu} \gamma_{1}(\mu)\right)\left(\frac{\mu}{u}\right)^{\frac{8}{3 \alpha}} d \mu, & \alpha \in\left(-\frac{2}{N}, 0\right) .
\end{array}\right.
$$

(3) The Hubble radius

$$
\frac{1}{a H}=\frac{3 b}{c} e^{-\frac{2 \varphi}{3}}\left(1+\sum_{n=1}^{\infty} b^{2 n} r_{n}(u) e^{-2 n \varphi}\right),
$$

where $r_{n}(u)$ are polynomials in $u$. For instance,

$$
r_{1}(u)=-\frac{4}{3} \gamma_{1}(u)
$$

(4) The variable $z(\varphi) \equiv-a h^{\prime} / h$

$$
z(\varphi)=-c e^{-\frac{\varphi}{3}}\left(1+\sum_{n=1}^{\infty} b^{2 n} z_{n}(u) e^{-2 n \varphi}\right)
$$

with $z_{n}(u)$ being polynomials in $u$. In particular,

$$
z_{1}(u)=-\left(\alpha u \gamma_{1}^{\prime}(u)+\frac{5}{3} \gamma_{1}(u)\right) .
$$



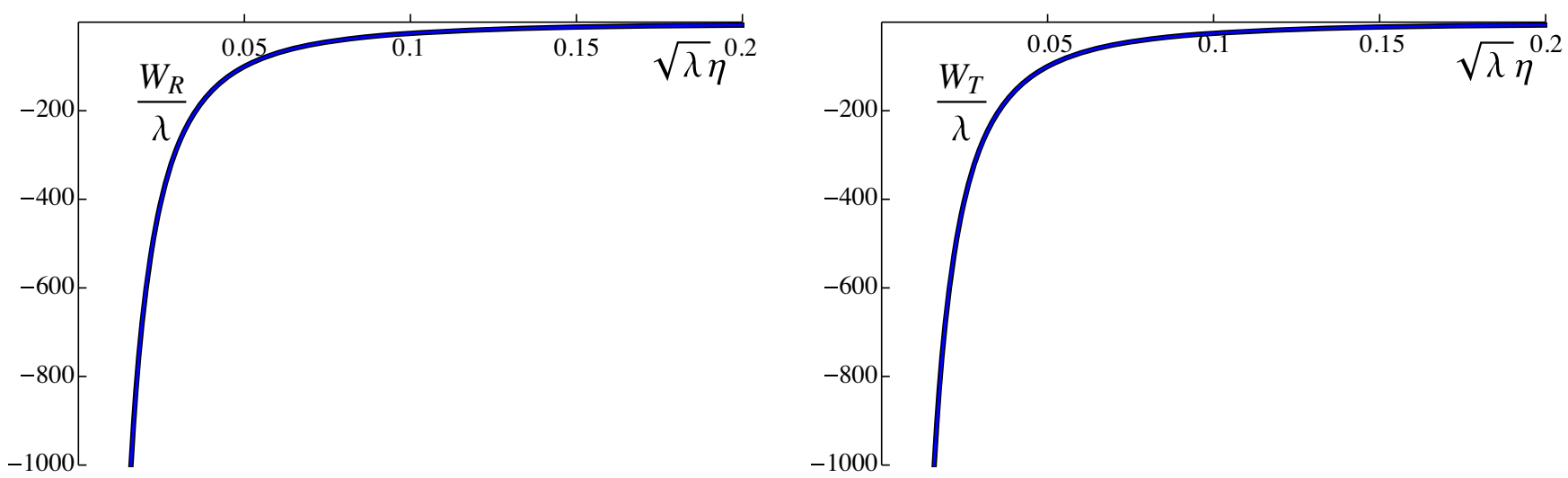

FIG. 9. Potentials $W_{R}(\eta)$ (left) and $W_{T}(\eta)$ (right) for the Starobinsky potential $v(\varphi)=\lambda\left(1-e^{-\frac{\varphi}{\sqrt{3}}}\right)^{2}$. The black lines show the numerical approximations, while the blue lines show the psi-series approximation [Eq. (138)]. Here we have used the rescaled variables $\sqrt{\lambda} \eta, \lambda^{-1} W_{R}, \lambda^{-1} W_{T}$, and the value $b=\hat{b}_{60} / \sqrt{\lambda}, \hat{b}_{60} \approx 2649.03$ obtained in Sec. IV A; $c$ is taken so that $a\left(\varphi_{\text {in }}\right)=1$ and $\eta\left(t^{*}\right)=0$ in agreement with Eq. (125).

From Eq. (125), we can derive the approximation

$\varphi \approx-\frac{3}{2} \log \left(\frac{2 c \eta}{3 b}\right)+\frac{3 b^{2}}{2}\left(\frac{2 c \eta}{3 b}\right)^{3} \eta_{1}\left(\left(\frac{2 c \eta}{3 b}\right)^{\frac{3 \alpha}{2}}\right)$.

By substituting Eq. (131) into Eq. (129), it is found that

$$
z(\eta) \approx-c\left(\frac{2 c}{3 b}\right)^{\frac{1}{2}} \eta^{\frac{1}{2}}\left[1+\eta^{3} \tilde{z}_{1}\left(\eta^{\alpha}\right)\right]
$$

where

$\tilde{z}_{1}\left(\eta^{\alpha}\right)=\frac{8 c^{3}}{27 b}\left[z_{1}\left(\left(\frac{2 c \eta}{3 b}\right)^{\frac{3 \alpha}{2}}\right)-\frac{1}{2} \eta_{1}\left(\left(\frac{2 c \eta}{3 b}\right)^{\frac{3 \alpha}{2}}\right)\right]$
Similarly, from Eqs. (123), (124), and (131), we have that

$$
a(\eta) \approx c\left(\frac{2 c}{3 b}\right)^{\frac{1}{2}} \eta^{\frac{1}{2}}\left[1+\eta^{3} \tilde{a}_{1}\left(\eta^{\alpha}\right)\right]
$$

with

$\tilde{a}_{1}\left(\eta^{\alpha}\right)=\frac{8 c^{3}}{27 b}\left[\frac{1}{3} \gamma_{1}\left(\left(\frac{2 c \eta}{3 b}\right)^{\frac{3 \alpha}{2}}\right)-\frac{1}{2} \eta_{1}\left(\left(\frac{2 c \eta}{3 b}\right)^{\frac{3 \alpha}{2}}\right)\right]$.

Thus, Eq. (132) leads to

$$
W_{R}(\eta) \approx \frac{1}{\eta^{2}}\left[-\frac{1}{4}+\eta^{3}\left(\alpha^{2} \eta^{2 \alpha} \tilde{z}_{1}^{\prime \prime}\left(\eta^{\alpha}\right)+\alpha(\alpha+6) \eta^{\alpha} \tilde{z}_{1}^{\prime}\left(\eta^{\alpha}\right)+9 \tilde{z}_{1}\left(\eta^{\alpha}\right)\right)\right], \quad \eta \rightarrow 0
$$

Analogously, from Eq. (134) one gets

$$
W_{T}(\eta) \approx \frac{1}{\eta^{2}}\left[-\frac{1}{4}+\eta^{3}\left(\alpha^{2} \eta^{2 \alpha} \tilde{a}_{1}^{\prime \prime}\left(\eta^{\alpha}\right)+\alpha(\alpha+6) \eta^{\alpha} \tilde{a}_{1}^{\prime}\left(\eta^{\alpha}\right)+9 \tilde{a}_{1}\left(\eta^{\alpha}\right)\right)\right], \quad \eta \rightarrow 0 .
$$

For example, for the Starobinsky model [Eq. (69)], from the first equation of Eq. (71), and Eqs. (124), (126), (130), (133), and (135)-(137), it is found that

$$
\begin{aligned}
& W_{R}(\eta) \approx-\frac{1}{4 \eta^{2}}-\frac{\lambda c^{3}}{3 b} \eta\left[\frac{2(\alpha+1)\left(6 \alpha^{2}+14 \alpha+7\right)}{3 \alpha+4}\left(\frac{2 c \eta}{3 b}\right)^{3 \alpha}-\frac{2(\alpha+2)\left(3 \alpha^{2}+14 \alpha+14\right)}{3 \alpha+8}\left(\frac{2 c \eta}{3 b}\right)^{\frac{3 \alpha}{2}}+\frac{7}{2}\right], \\
& W_{T}(\eta) \approx-\frac{1}{4 \eta^{2}}+\frac{\lambda c^{3}}{3 b} \eta\left[\frac{2(\alpha+1)}{3 \alpha+4}\left(\frac{2 c \eta}{3 b}\right)^{3 \alpha}-\frac{4(\alpha+2)}{3 \alpha+8}\left(\frac{2 c \eta}{3 b}\right)^{\frac{3 \alpha}{2}}+\frac{1}{2}\right] .
\end{aligned}
$$


The potentials (138) are plotted in Fig. 9, where $\alpha=\frac{1}{\sqrt{3}}$, $b=b_{60}$ is the value obtained in Sec. IV A for the Starobinsky potential and $c$ is taken such that $a\left(\varphi_{\text {in }}\right)=1$.

We notice that, as was found in Ref. [11], the common dominant term near the singularity $\eta=0$ of the potentials (118), (119), (136), and (137) coincides with the critical central singular attractive potential, allowing the fall to the center of a quantum particle.

Finally, we point out that we may also use our psi series in the variable $\varphi$ starting from the Mukhanov-Sasaki equation (99) with the potential $W_{\alpha}$ expressed as a function of $\varphi$. However, as is shown in Eq. (2.27) of Ref. [11], the function $W_{\alpha}(\varphi)$ is rather involved, so we do not find any advantage in using our psi series in that way.

\section{CONCLUSIONS}

In this paper, we have developed a method to determine psi-series formal solutions of single-field inflation models [Eqs. (1) and (2)] during the kinetic dominance period. The scheme is based on the Hamilton-Jacobi formalism of inflaton models [28-30] and provides psi series depending on the inflaton field. The method has been applied to models with polynomial-exponential potential functions [Eq. (4)] (two particularly important examples are the quadratic potential [Eq. (48)] and the Higgs potential [Eq. (51)]) and to models with generalized Starobinsky potential functions Eq. (5) (including the standard Starobinsky potential [Eq. (69)]). We have also proved that there exist psi series near the singularity for several physically relevant quantities such as the scale factor, the conformal time, and the Hubble radius. The explicit form of the first two terms of these expansions has been given.

We have found that truncations of these psi series can be used to determine the value of the inflaton field at the initial moment of the inflation period, and to include the effect of the KD period to estimate the amount of inflation. Furthermore, we have shown that psi series can be applied to determine explicit corrections depending on the inflaton field to the dominant term of the potentials of the MukanovSasaki equation for both curvature and tensor perturbations.

\section{ACKNOWLEDGMENTS}

The financial support of the Spanish Ministerio de Ciencia, Innovación y Universidades under Projects No. FIS2015-63966-P and No. PGC2018-094898-B-I00 is gratefully acknowledged. We thank Professor Gabriel Alvarez Galindo for fruitful discussions.
[1] A. Starobinsky, A new type of isotropic cosmological models without singularity, Phys. Lett. 91B, 99 (1980).

[2] A. H. Guth, Inflationary universe: A possible solution to the horizon and flatness problems, Phys. Rev. D 23, 347 (1981).

[3] A. D. Linde, A new inflationary universe scenario: A possible solution of the horizon, flatness, homogeneity, isotropy and primordial monopole problems, Phys. Lett. 108B, 389 (1982).

[4] A. D. Linde, Initial conditions for inflation, Phys. Lett. 162B, 281 (1985).

[5] V. Mukhanov, Physical Foundations of Cosmology (Cambridge University Press, Cambridge, England, 2005).

[6] D. Baumann, TASI Lectures on inflation, arXiv:0907.5424.

[7] J. Martin, The theory of Inflation, in Proceedings of the International School of Physics "Enrico Fermi" 200, 155 (2020).

[8] A. Lasenby and C. Doran, Closed universes, de Sitter space, and inflation, Phys. Rev. D 71, 063502 (2005).

[9] W. Handley, S. Brechet, A. Lasenby, and M. P. Hobson, Kinetic initial conditions for inflation, Phys. Rev. D 89, 063505 (2014).

[10] W. Handley, A. Lasenby, and M. Hobson, Logolinear series expansions with applications to primordial cosmology, Phys. Rev. D 99, 123512 (2019).

[11] C. Destri, H. de Vega, and N. G. Sanchez, Preinflationary and inflationary fast-roll eras and their signatures in the low CMB multipoles, Phys. Rev. D 81, 063520 (2010).
[12] P. J. Steinhardt and M. S. Turner, Prescription for successful new inflation, Phys. Rev. D 29, 2162 (1984).

[13] E. D. Stewart and D. H. Lyth, A more accurate analytic calculation of the spectrum of cosmological perturbations produced during inflation, Phys. Lett. B 302, 171 (1993).

[14] A. R. Liddle, P. Parsons, and J. D. Barrow, Formalizing the slow-roll approximation in inflation, Phys. Rev. D 50, 7222 (1994).

[15] J. E. Lidsey, A. R. Liddle, E. W. Kolb, E. J. Copeland, T. Barreiro, and M. Abney, Reconstructing the inflaton potential-an overview, Rev. Mod. Phys. 69, 373 (1997).

[16] B. A. Bassett, S. Tsujikawa, and D. Wands, Inflation dynamics and reheating, Rev. Mod. Phys. 78, 537 (2006).

[17] A. Linde, Inflationary cosmology, in Inflationary Cosmology, Lecture Notes in Physics Vol. 738 (Springer, New York, 2008), pp. 1-54.

[18] S. Weinberg, Cosmology (Oxford University Press, New York, 2008).

[19] D. Boyanovsky, C. Destri, H. de Vega, and N. Sanchez, The effective theory of inflation in the standard model of the universe and the $\mathrm{cmb}+\mathrm{lss}$ data analysis, Int. J. Mod. Phys. A 24, 3669 (2009).

[20] W. I. J. Haddadin and W. J. Handley, Rapid numerical solutions for the Mukhanov-Sazaki equation, arXiv:1809 .11095 . 
[21] L. Hergt, W. Handley, M. Hobson, and A. Lasenby, Constraining the kinetically dominated universe, Phys. Rev. D 100, 023501 (2019).

[22] L. Hergt, W. Handley, M. Hobson, and A. Lasenby, Case for kinetically dominated initial conditions for inflation, Phys. Rev. D 100, 023502 (2019).

[23] M. Tabor and J. Weiss, Analytic structure of the Lorenz system, Phys. Rev. A 24, 2157 (1981).

[24] E. Hille, Ordinary Differential Equations in the Complex Domain (Courier Corporation, John Wiley \& Sons, New York, USA, 1976).

[25] Y. Chang, J. Greene, M. Tabor, and J. Weiss, The analytic structure of dynamical systems and self-similar natural boundaries, Physica (Amsterdam) 8D, 183 (1983).

[26] J. Fournier, G. Levine, and M. Tabor, Singularity clustering in the Duffing oscillator, J. Phys. A 21, 33 (1988).

[27] V. E. Tarasov, Psi-series solution of fractional GinzburgLandau equation, J. Phys. A 39, 8395 (2006).

[28] D. Salopek and J. Bond, Nonlinear evolution of longwavelength metric fluctuations in inflationary models, Phys. Rev. D 42, 3936 (1990).
[29] A. R. Liddle and D. H. Lyth, Cosmological Inflation and Large-Scale Structure (Cambridge University Press, Cambridge, England, 2000).

[30] G. Álvarez, L. Martínez Alonso, E. Medina, and J. L. Vázquez, Separatrices in the Hamilton-Jacobi formalism of inflaton models, J. Math. Phys. (N.Y.) 61, 043501 (2020).

[31] D. Baumann, Cosmology Part III, Lecture Notes, Mathematical Tripos Classes (Cambridge, 2012), http://docplayer .net/156231655-Cosmology-part-iii-mathematical-triposdaniel-baumann-sec-380-000-yrs-billion-yrs.html.

[32] S. Dodelson, Modern Cosmology (Academic Press, New York, USA, 2003), ISBN 9780122191411.

[33] A. Starobinskii, The perturbation spectrum evolving from a nonsingular initially de-Sitter cosmology and the microwave background anisotropy, Sov. Astron. Lett. 9, 302 (1983), http://articles.adsabs.harvard.edu/pdf/1983SvAL... .9..302S.

[34] B. Whitt, Fourth-order gravity as general relativity plus matter, Phys. Lett. 145B, 176 (1984).

[35] E. T. Bell, Exponential Polynomials, Ann. Math. 35, 258 (1934). 